\title{
SU(3) Anderson impurity model: A numerical renormalization group approach exploiting non-Abelian symmetries
}

\author{
Cătălin Paşcu Moca \\ BME-MTA Exotic Quantum Phase Group, Institute of Physics, Budapest University of Technology and Economics, H-1521 Budapest, \\ Hungary and Department of Physics, University of Oradea, 410087, Oradea, Romania
}

Arne Alex and Jan von Delft

Physics Department, Arnold Sommerfeld Center for Theoretical Physics and Center for NanoScience, Ludwig-Maximilians-Universität München, D-80333 München, Germany

Gergely Zaránd

BME-MTA Exotic Quantum Phase Group, Institute of Physics, Budapest University of Technology and Economics, H-1521 Budapest, Hungary

(Received 3 August 2012; published 19 November 2012)

\begin{abstract}
We show how the density-matrix numerical renormalization group method can be used in combination with nonAbelian symmetries such as $\mathrm{SU}(N)$. The decomposition of the direct product of two irreducible representations requires the use of a so-called outer multiplicity label. We apply this scheme to the SU(3) symmetrical Anderson model, for which we analyze the finite size spectrum, determine local fermionic, spin, superconducting, and trion spectral functions, and also compute the temperature dependence of the conductance. Our calculations reveal a rich Fermi liquid structure.
\end{abstract}

DOI: 10.1103/PhysRevB.86.195128

PACS number(s): 72.10.Fk, 73.63.Kv

\section{INTRODUCTION}

Quantum impurity models, describing a quantum system with a small number of discrete states, the impurity, coupled to a continuous bath of fermionic or bosonic excitations, arise in a variety of contexts. A particularly important example is the Anderson impurity model, ${ }^{1}$ relevant for describing magnetic moments in metals, transport through quantum dots, and for the treatment of correlated lattice models using dynamical mean-field theory. While the standard version of this model has SU(2) spin symmetry in the absence of a magnetic field, generalizations to settings with higher symmetry have also been studied. The $\mathrm{SU}(N)$ generalization of the Anderson model emerged first in the context of heavy Fermion systems, ${ }^{2}$ where large $N$ expansions proved to be an efficient way to model and describe magnetic atoms with orbital degeneracy. Studying these models in detail is not only useful in the context of heavy fermion systems, but it also represents the first step to understand the behavior of correlated cold atomic gases with $\mathrm{SU}(N)$ symmetrical interactions. ${ }^{3,4}$

The $\mathrm{SU}(N)$ Anderson model can also be realized in a controlled way. In particular, the SU(4) model has been realized in various mesoscopic structures including carbon nanotubes, ${ }^{5,6}$ vertical quantum dots, ${ }^{7}$ and more recently in the originally proposed double dot structures. ${ }^{8}$ Similarly, the SU(3) Anderson model could also be realized with quantum dot structures, though the proposed setup is maybe somewhat more complicated. ${ }^{9}$

The $\mathrm{SU}(N)$ Anderson model is defined in terms of $N$ local orbitals embedded in a conduction electron sea. Its Hamiltonian can be written in terms of the corresponding creation operators, $d_{\alpha}^{\dagger}(\alpha=1, \ldots, N)$ and the number operator $\hat{n}=\sum_{\alpha} d_{\alpha}^{\dagger} d_{\alpha}$ as

$$
\begin{gathered}
H=\varepsilon_{d} \hat{n}+\frac{U}{2} \hat{n}(\hat{n}-1)+V \sum_{\alpha}\left(d_{\alpha}^{\dagger} \psi_{\alpha}(0)+\text { H.c. }\right) \\
+H_{\text {chan }}\left[\psi, \psi^{\dagger}\right] .
\end{gathered}
$$

Here $\varepsilon_{d}$ and $U$ denote the position of the local orbital and the strength of interaction on it, respectively, and the level $d^{\dagger}$ hybridizes locally with the fermions at its position, destroyed by $\psi_{\alpha}(0)$. The last term of the Hamiltonian describes the kinetic energy of the conduction electrons. It generates the dynamics of the field $\psi_{\alpha}(0)$, and amounts to a broadening of the "atomic" level $\varepsilon_{d}$.

In the present paper we show on the prototypical example of the SU(3) symmetrical Anderson model how the numerical renormalization group ${ }^{10,11}$ (NRG) of Wilson, one of the most versatile and reliable tools for treating quantum impurity models, can be adapted to fully take advantage of nonAbelian symmetries to reduce computational costs. Within Wilson's procedure, one rewrites (1) as the Hamiltonian of a semi-infinite chain, and diagonalizes it iteratively. ${ }^{10}$ Using symmetries in the course of this diagonalization procedure is crucial: It allows computer memory to be used efficiently, and enables one to reach the required numerical accuracy on relatively standard computers with reasonable run times. Equation (1) obviously possesses an $\mathrm{SU}(N) \times \mathrm{U}(1)$ symmetry corresponding to rotations in spin space and overall charge conservation. Here, we shall focus on the $N=3$ case, classify states and observables while exploiting these symmetries, and determine the spectral functions of several local observables.

In an earlier work, a general framework has been set up and implemented to handle an arbitrary number of non-Abelian symmetries dynamically. ${ }^{12}$ This formulation allowed us to build an open-access flexible density-matrix NRG (DM-NRG) code. ${ }^{13}$ However, in Ref. 12 we considered only combinations of certain rather simple symmetries such as charge and spin $\mathrm{SU}(2)$ symmetries, $\mathrm{Z}_{2}$ or $\mathrm{U}(1)$ symmetries. In a group theoretical sense, these are simpler than $\operatorname{SU}(N>2)$ and some other discrete or Lie groups. For SU(2), irreducible representations (irreps) are labeled by the size of the spin. When "adding" two SU(2) spins, say $S_{1}$ and $S_{2}$, each possible 
total spin $S_{3}$ that satisfies the angular momentum addition rule $\left|S_{1}-S_{2}\right| \leqslant S_{3} \leqslant S_{1}+S_{2}$ can be obtained in precisely one way. More technically, in the decomposition of the direct product of two $S U(2)$ irreps labeled by spins $S_{1}$ and $S_{2}$ into a direct sum of irreps, the number of times $n_{S_{1} S_{2} ; S_{3}}$ that any irrep labeled by spin $S_{3}$ occurs in the direct sum, the so-called "outer multiplicity" is either 0 or 1 . This is, however, not true for $\operatorname{SU}(N>2)$ : The decomposition of the direct product $\Gamma_{1} \otimes \Gamma_{2}$ of two $\mathrm{SU}(N)$ irreps into a direct sum can contain irreps with outer multiplicity $n_{\Gamma_{1} \Gamma_{2} ; \Gamma_{3}}$ larger than 1 , in other words, there may be $n_{\Gamma_{1} \Gamma_{2} ; \Gamma_{3}}$ inequivalent ways to construct the irrep $\Gamma_{3}$ from $\Gamma_{1}$ and $\Gamma_{2}$. Correspondingly, the Clebsch-Gordan coefficients have a more complicated structure in this case, and the Wigner-Eckart theorem, extensively used in the DM-NRG calculations, becomes also somewhat more complicated. Here we show how a general framework can be constructed to deal with this case, ${ }^{14}$ and demonstrate it on the specific example of the SU(3) Anderson model.

We note that another general approach towards exploiting non-Abelian symmetries such as $\mathrm{SU}(N)$ or $\operatorname{Sp}(N)$ within NRG, and more generally for tensor network methods, has recently been published by Weichselbaum. ${ }^{15}$ It is formulated in the language of matrix product states, and may be regarded as complementary to our own, which is phrased within the more traditional formulation of NRG. We emphasize, though, that both approaches are fully equivalent, in that precisely the same NRG assumptions and approximations are made in both; they differ only in the data structures used for internal bookkeeping in the numerical codes. Their relation is briefly sketched in Appendix D.

Our DM-NRG calculations require explicit knowledge of Clebsch-Gordan coefficients. Whereas these are known in closed form for $\mathrm{SU}(2)$, this is not the case for $\mathrm{SU}(N>2)$. However, an efficient numerical algorithm for their evaluation has recently been developed, ${ }^{16}$ which we use here.

This paper is structured as follows: In Sec. II we outline our approach for exploiting non-Abelian symmetries in DM-NRG calculations. In Sec. III we apply it to the SU(3) Anderson model; in particular, we present results for the conductance through a quantum dot described by this model, and for various local spectral functions. Our conclusions in Sec. IV are followed by four Appendixes that summarize some basic facts of $\mathrm{SU}(N)$ representation theory, and some recursion formulas involving Clebsch-Gordan coefficients, respectively.

\section{DM-NRG WITH NON-ABELIAN SYMMETRIES}

As stated in the introduction, the formalism presented in Ref. 12 applies only for a special (although relatively large) class of symmetries. Therefore, let us review here the most important formulas and the structure of the NRG calculations for the more general case, where so-called outer multiplicities are also considered. ${ }^{17}$

\section{A. Local symmetries on the Wilson chain}

Let us start by first discussing the general structure of the symmetries of the Wilson chain. The first step in Wilson's procedure of solving a quantum impurity problem is to perform a Gram-Schmidt orthogonalization and rewrite the
Hamiltonian in a "tridiagonal" form, ${ }^{11}$

$$
H=\mathcal{H}_{0}+\sum_{n=0}^{\infty}\left(\tau_{n, n+1}+\mathcal{H}_{n+1}\right) .
$$

Here $\mathcal{H}_{0}$ contains the local, interacting part of the Hamiltonian, while the rest of the chain represents the conduction electron (bath) degrees of freedom, coupled to it. The on-site terms $\mathcal{H}_{n+1}$ are many times missing; they typically appear for more sophisticated electronic densities of states and can also account for superconducting correlations. In our case, one could take, for example,

$$
\mathcal{H}_{0}=\varepsilon_{d} \hat{n}+\frac{U}{2} \hat{n}(\hat{n}-1)+\tilde{V} \sum_{\alpha}\left(d_{\alpha}^{\dagger} f_{\alpha}^{[0]}+\text { h.c. }\right),
$$

as $\mathcal{H}_{0}$, with $f_{\alpha}^{[0]} \sim \psi_{\alpha}(0)$ a properly normalized on-site fermion, and the hopping terms $\tau_{n, n+1}$ would read

$$
\tau_{n, n+1}=\sum_{\alpha} h^{[n]}\left(f_{\alpha}^{[n] \dagger} f_{\alpha}^{[n+1]}+\text { h.c. }\right) .
$$

Here $f_{\alpha}^{[n+1]}$ annihilates a fermion of SU(3) spin $\alpha$ at site [n], and the hopping amplitudes $h^{[n]}$ decay exponentially along the chain, thereby leading to energy scale separation. Equation (2) is then diagonalized iteratively using the recursive relation,

$$
H_{n}=H_{n-1}+\tau_{n-1, n}+\mathcal{H}_{n}
$$

In the following, we shall assume that $H$ (and $H_{n}$ ) are invariant under the direct product of $n_{S}$ symmetry groups,

$$
G=\mathcal{G}_{1} \times \mathcal{G}_{2} \times \cdots \times \mathcal{G}_{n_{S}} .
$$

This means that for any group element $g_{\lambda} \in \mathcal{G}_{\lambda}$, with $\lambda=$ $1, \ldots, n_{S}$, there exists a unitary operator $\mathcal{U}_{\lambda}\left(g_{\lambda}\right)$ on the Fock space, leaving $H$ invariant. Here we do not need to make much restriction on the groups: Our considerations hold for any group which acts on the chain locally at each lattice site $n$,

$$
\mathcal{U}(g)=\prod_{\lambda=1}^{n_{S}} \mathcal{U}_{\lambda}\left(g_{\lambda}\right)=\prod_{\lambda=1}^{n_{S}} \prod_{n} \mathcal{U}_{\lambda, n}\left(g_{\lambda}\right),
$$

and for which the Wigner-Eckart theorem holds. ${ }^{18}$ Given the above group structure, we can group all eigenstates and also operators into multiplets, each of which transforms according to a certain representation of $\mathcal{G}$,

$$
\Gamma \equiv\left\{\Gamma^{1}, \Gamma^{2}, \ldots, \Gamma^{n_{S}}\right\} \leftrightarrow \Gamma^{1} \otimes \Gamma^{2} \otimes \cdots \otimes \Gamma^{n_{S}} .
$$

The "quantum numbers" $\Gamma^{\lambda}$, which label the various irreducible representations (irreps) occurring in $\Gamma$, can be spin labels, charges, or can label different irreps of some point group. States within a multiplet are then labeled by a set of internal indices, $\gamma=\left\{\gamma^{1}, \gamma^{2}, \ldots, \gamma^{n_{S}}\right\}$, with the internal labels running from $1 \leqslant \gamma^{\lambda} \leqslant \operatorname{dim}\left(\Gamma^{\lambda}\right)$. A given multiplet $i$ that transforms according to the representation $\Gamma_{i}$ thus consists of

$$
\operatorname{dim}(i) \equiv \operatorname{dim}\left(\Gamma_{i}\right)=\prod_{\lambda=1}^{n_{S}} \operatorname{dim}\left(\Gamma_{i}^{\lambda}\right)
$$

degenerate states. 
States belonging to a product of two representations, $\Gamma_{1}^{\lambda} \otimes \Gamma_{2}^{\lambda}$, often appear in the calculations. As outlined in the introduction, similar to spins, these can be decomposed into irreps. However, one irrep may appear several times in this decomposition,

$$
\Gamma_{s}^{\lambda} \otimes \Gamma_{p}^{\lambda} \rightarrow \ldots \oplus \underbrace{\Gamma_{q}^{\lambda} \oplus \ldots \oplus \Gamma_{q}^{\lambda}}_{n_{s, p ; q}^{\lambda} \text { times }} \oplus \ldots
$$

Accordingly, in the most general case, the resulting states $\Gamma_{s}^{\lambda} \otimes \Gamma_{p}^{\lambda} \rightarrow \Gamma_{q}^{\lambda}$ must be labeled by a so-called "outer multiplicity" label, $\alpha_{\lambda}=1, \ldots, n_{s, p ; q}^{\lambda}$. Correspondingly, properly transforming multiplets may be constructed from the product of two multiplets as

$$
|\Gamma, \gamma\rangle_{\alpha}=\sum_{\gamma_{1}, \gamma_{2}}\left(\Gamma_{1}, \gamma_{1} ; \Gamma_{2}, \gamma_{2} \mid \Gamma, \gamma\right)_{\alpha}\left|\Gamma_{1}, \gamma_{1}\right\rangle \otimes\left|\Gamma_{2}, \gamma_{2}\right\rangle,
$$

where $\alpha \equiv\left\{\alpha_{\lambda}\right\}$ denotes the composite multiplicity label, and the generalized Clebsch-Gordan coefficients are defined as

$$
\left(\Gamma_{1}, \gamma_{1} ; \Gamma_{2}, \gamma_{2} \mid \Gamma, \gamma\right)_{\alpha} \equiv \prod_{\lambda=1}^{n_{S}}\left(\Gamma_{1}^{\lambda}, \gamma_{1}^{\lambda} ; \Gamma_{2}^{\lambda}, \gamma_{2}^{\lambda} \mid \Gamma^{\lambda}, \gamma^{\lambda}\right)_{\alpha_{\lambda}} .
$$

The outer multiplicity also appears in the Wigner-Eckart theorem. The latter states that the matrix elements of an operator multiplet (i.e., a set of operators $\left\{A_{\Gamma_{A}, \gamma_{A}}\right\}$ transforming under transformations $\mathcal{U}(g)$ as a multiplet $\left.\Gamma_{A}\right)$ are determined almost entirely by representation theory, and can be expressed in terms of the Clebsch-Gordan coefficients as

$$
\begin{aligned}
& \left\langle i, \Gamma_{i}, \gamma_{i}\left|A_{\Gamma_{A}, \gamma_{A}}\right| j, \Gamma_{j}, \gamma_{j}\right\rangle \\
& \quad=\sum_{\alpha}\left(\Gamma_{A}, \gamma_{A} ; \Gamma_{j}, \gamma_{j} \mid \Gamma_{i}, \gamma_{i}\right)_{\alpha}^{*}\langle i\|A\| j\rangle_{\alpha} .
\end{aligned}
$$

Here multiplets $i$ and $j$ transform according to the irreps, $\Gamma_{i}$ and $\Gamma_{j}$. Thus, according to the Wigner-Eckart theorem, all matrix elements are determined by only a few reduced matrix elements, $\langle i\|A\| j\rangle_{\alpha}$, labeled just by the outer multiplicity labels, $\alpha$ characterizing how many times the representation $\Gamma_{i}$ appears in the product of $\Gamma_{j}$ and $\Gamma_{A}$. For many commonly used symmetries as $\mathrm{SU}(2)$, for example, the outer multiplicity is just always one and the label $\alpha$ can be dropped. However, it is needed for, for example, $\mathrm{SU}(N \geqslant 3)$ or even for cubic point groups.

\section{B. Wilson's NRG with symmetries}

In course of the NRG procedure, one diagonalizes Eq. (2) iteratively. The eigenstates of the Hamiltonian $H_{n}$ of a chain of length $n$ can be grouped into multiplets, with each multiplet $i$ transforming according to a certain representation $\Gamma_{i}=$ $\left\{\Gamma_{i}^{1}, \ldots, \Gamma_{i}^{n_{S}}\right\}$. Having computed the approximate eigenstates (block states) $\left|i, \Gamma_{i}, \gamma_{i},\right\rangle^{[n-1]}$ of $H_{n-1}$, one proceeds to construct eigenstates of $H_{n}$. To do that, one first appends to the chain the multiplets $\left\{\left|\mu, \Gamma_{\mu}^{\text {loc }}, \gamma_{\mu}^{\text {loc }}\right\rangle\right\}$, spanning local Hilbert space at site $n$, and then constructs properly transforming multiplets $\left\{\left|u, \Gamma_{u}, \gamma_{u}\right\rangle_{\alpha_{u}}^{[n]}\right\}$ by making use of the Clebsch-Gordan coefficients [Eq. (11)]:

$$
\left|\mu, \Gamma_{\mu}^{\mathrm{loc}}, \gamma_{\mu}^{\mathrm{loc}}\right\rangle \otimes\left|i, \Gamma_{i}, \gamma_{i}\right\rangle^{[n-1]} \rightarrow\left|u, \Gamma_{u}, \gamma_{u}\right\rangle_{\alpha_{u}}^{[n]} .
$$

Notice that a new multiplet $u$ now also carries an outer multiplicity label $\alpha_{u}$ : This specifies the representation according to which $\Gamma_{u}$ has been produced from $\Gamma_{i}$ and $\Gamma_{\mu}$. The advantage of using these states is that $H_{n}$ is diagonal both in $\Gamma_{u}$ and in the internal labels $\gamma_{u}$. Therefore, it is sufficient to compute only the corresponding irreducible matrix elements $\left\langle u\left\|H_{n}\right\| v\right\rangle^{[n]}$ in each symmetry sector (block) separately, and diagonalize $H_{n}$ sectorwise by a unitary transformation to obtain the corresponding new eigenstates,

$$
\left|u, \Gamma_{u}, \gamma_{u}\right\rangle_{\alpha_{u}}^{[n]} \rightarrow\left|\tilde{i}, \Gamma_{\tilde{i}}, \gamma_{\tilde{i}}\right\rangle^{[n]} .
$$

As explained in Appendix D, this iterative procedure leads to a matrix product state (MPS) with a peculiar structure, reflecting the symmetry of the Hamiltonian.

The most difficult part in the procedure above is to determine the matrix elements $\left\langle u\left\|H_{n}\right\| v\right\rangle^{[n]}$. These can be constructed by noticing that each state $u$ and $v$ has been constructed from the eigenstates of $H_{n-1}$ and $\mathcal{H}_{n}, i, \mu \rightarrow u$, and $j, v \rightarrow v$, and therefore

$$
\left\langle u\left\|H_{n}\right\| v\right\rangle^{[n]}=\delta_{u, v}\left(E_{i}^{n-1}+\epsilon_{\mu}^{n}\right)+\left\langle u\left\|\tau_{n-1, n}\right\| v\right\rangle^{[n]},
$$

with $\epsilon_{\mu}^{n}$ being the eigenenergy of $\mathcal{H}_{n}$. The matrix elements of $\tau_{n-1, n}$ can be worked out by assuming that the hopping part consists of some fermionic or bosonic creation operators $C_{a, \Gamma_{a}, \gamma_{a}}^{[n]}$, transforming again according to some irreps $\Gamma_{a}$,

$$
\tau_{n-1, n}=\sum_{a}\left[h_{a}^{[n-1]} \sum_{\gamma_{a}} C_{a, \Gamma_{a}, \gamma_{a}}^{[n-1]}\left(C_{a, \Gamma_{a}, \gamma_{a}}^{[n]}\right)^{\dagger}+\text { h.c. }\right] .
$$

Here $a$ labels the different "hopping operators," and $h_{a}^{[n-1]}$ the corresponding hopping amplitudes. Notice the somewhat unusual way this hopping term is written: $C_{a, \Gamma_{a}, \gamma_{a}} \leftrightarrow f^{\dagger}$ is a "creation operator," which transforms according to the representation $\Gamma_{a}$, while $\left(C_{a, \Gamma_{a}, \gamma_{a}}\right)^{\dagger} \leftrightarrow f$ is an "annihilation operator," transforming according to the conjugate representation $\Gamma_{a}{ }^{*}$. We remark that for charge $\mathrm{SU}(2)$ symmetry, for example, the "creation" operator multiplet $C_{a, \Gamma_{a}, \gamma_{a}}$ is a Nambu spinor, and contains both $f$ and $f^{\dagger}$ operators. ${ }^{20}$ The number of hopping operators may depend on the symmetry used: For a chain of spin $1 / 2$ fermions treated in terms of $S U(2) \times U(1)$ symmetry, for example, one has a single hopping operator of spin $1 / 2$ and charge 1 , while if only the charge symmetry is used then one has two hopping operators of charge 1, corresponding to the spin-up and spin-down directions. In our example of the SU(3) Anderson model we have a single hopping operator, and $C_{\Gamma_{a}, \gamma_{a}}^{[n]} \leftrightarrow\left\{f_{\alpha}^{[n] \dagger}\right\}$. Assuming then that the reduced matrix elements of the creation operators acting on site $n-1$ of the chain, $\left\langle u\left\|C_{a}^{[n-1]}\right\| v\right\rangle_{\alpha}^{[n-1]}$, and those of the local creation operators at the added site, $\left\langle v\left\|C_{a}^{[n]}\right\| \mu\right\rangle_{\beta}$ are known, one can use the Wigner-Eckart theorem to express $\left\langle u\left\|\tau_{n-1, n}\right\| v\right\rangle^{[n]}$ as

$$
\begin{aligned}
\left\langle u\left\|\tau_{n-1, n}\right\| v\right\rangle^{[n]}= & \delta_{\Gamma_{u}, \Gamma_{v}} \sum_{a, \alpha, \beta} h_{a}^{[n-1]}\left\langle i\left\|C_{a}^{[n-1]}\right\| j\right\rangle_{\alpha}^{[n-1]} \\
& \times\left\langle v\left\|C_{a}^{[n]}\right\| \mu\right\rangle_{\beta}^{*} D(a, \alpha, \beta ; u, v)+\text { h.c. } \\
& (u \leftarrow \mu, i ; v \leftarrow v, j) .
\end{aligned}
$$

Here the outer multiplicity labels $\alpha(\beta)$ label inequivalent ways in which $\Gamma_{i}\left(\Gamma_{\nu}\right)$ appear in the product $\Gamma_{a} \otimes \Gamma_{j}\left(\Gamma_{a} \otimes \Gamma_{\mu}\right)$. The coefficients $D(a, \alpha, \beta ; u, v)$ can be expressed in terms of Clebsch-Gordan coefficients, and are given by Eq. (C3) in 
Appendix C. Similar expressions hold for the matrix elements of "block operators" $(A)$, acting somewhere on the first $n-1$ sites of the chain, and those of "local operators" $A^{[n]}$, acting only on the last site of the chain. ${ }^{19}$ For a block operator we have, for example,

$$
\langle u\|A\| v\rangle_{\beta}^{[n]}=\sum_{\alpha}\langle i\|A\| j\rangle_{\alpha}^{[n-1]} F(\alpha, \beta ; u, v) \delta_{\mu, \nu},
$$

while for the local operators the following equation holds,

$$
\left\langle u\left\|A^{[n]}\right\| v\right\rangle_{\beta}^{[n]}=\sum_{\alpha}\left\langle\mu\left\|A^{[n]}\right\| v\right\rangle_{\alpha} K(\alpha, \beta ; u, v) \delta_{i, j},
$$

with the coefficients $F(\alpha, \beta ; u, v)$ and $K(\alpha, \beta ; u, v)$ given in Appendix C. Here again, the outer multiplicity labels $\beta$ label inequivalent ways in which $\Gamma_{u}$ appears in $\Gamma_{A} \otimes \Gamma_{v}$, while $\alpha$ labels similarly inequivalent ways how $\Gamma_{i}\left(\Gamma_{\mu}\right)$ can be constructed from $\Gamma_{A}$ and $\Gamma_{j}\left(\Gamma_{A}\right.$ and $\left.\Gamma_{v}\right)$. Similar to $D$, the coefficients $F$ and $K$ are again determined only by symmetry, and can be expressed in terms of Clebsch-Gordan coefficients. As a last step of the iteration, the reduced matrix elements $\left\langle u\left\|A^{[n]}\right\| v\right\rangle_{\beta}^{[n]}$ and $\langle u\|A\| v\rangle_{\beta}^{[n]}$ need be transformed to the new basis, $\left|\tilde{i}, \Gamma_{\tilde{i}}, \gamma_{i}\right\rangle$. This is performed by using precisely the same unitary block transformations as the ones used to diagonalize the Hamiltonian $H_{n}$ [Eq. (15)], without affecting the outer multiplicity labels $\beta$.

Wilson's diagonalization procedure can be carried out then based upon the equations above: In a given iteration, one takes the lowest lying states of iteration $[n-1]$ and their matrix elements $\left\langle i\left\|C_{a}^{[n-1]}\right\| j\right\rangle_{\beta}^{[n-1]}$, and computes from these and from the matrix elements $\left\langle\mu\left\|C_{a}^{[n]}\right\| v\right\rangle_{\beta}$ the Hamiltonian $\left\langle u\left\|H_{n}\right\| v\right\rangle^{[n]}$. Then diagonalizing $\left\langle u\left\|H_{n}\right\| v\right\rangle^{[n]}$, one obtains low-lying eigenstates of $H_{n}$ and determines their matrix elements $\left\langle\tilde{i}\left\|C_{a}^{[n]}\right\| \tilde{j}\right\rangle_{\beta}^{[n]}$. Continuing this procedure for larger and larger values $n$, one obtains better and better approximations for the ground state of $H=H_{n \rightarrow \infty}$ and the lowest lying eigenstates.

\section{FDM-NRG approach}

So far, we discussed essentially Wilson's original NRG approach in case of general symmetries. In practice, however, one often needs to go beyond Wilson's RG and use the so-called DM-NRG method, ${ }^{21}$ whereby a forward NRG run is first performed to obtain the density matrix (DM) of the system, and then a backward NRG run is made to compute physical observables. Moreover, to satisfy spectral sum rules, a complete basis $\operatorname{set}^{25,26}$ has to be used, as first implemented in the context of DM-NRG in Refs. 22 and 23. In the full density-matrix NRG approach (FDM-NRG) of Ref. 23, the full density matrix of the entire chain is expressed in the complete basis, which yields an improved treatment of finite-temperature properties. Let us now briefly discuss how symmetries can be implemented in the FDM-NRG approach. (For a complimentary formulation of the same strategy using matrix product states, see Ref. 15.)

First, to satisfy the necessary completeness relations, we consider a chain of $N$ sites and introduce "environment" states $e$ for each state discarded in iteration $[n](i \in D),{ }^{22,23}$

$$
\left|i, \Gamma_{i} \gamma_{i}\right\rangle^{[n]} \rightarrow\left|i, \Gamma_{i} \gamma_{i} ; e_{n}\right\rangle^{[n]}
$$

Here the states $e_{n}$ form an orthonormal basis for the remaining $N-n$ sites of the chain, and their internal structure is irrelevant for the remaining discussion.

The states [Eq. (20)] form a complete basis on the Wilson chain, ${ }^{25,26}$ and can be used to construct the density operator as follows, ${ }^{23}$

$$
\begin{aligned}
\varrho & =\sum_{n=0}^{N} \varrho^{[n]}, \\
\varrho^{[n]} & \equiv \sum_{i \in D, e_{n}} \sum_{\gamma_{i}} \frac{e^{-\beta E_{i}^{n}}}{\mathcal{Z}}\left|i, \Gamma_{i}, \gamma_{i} ; e_{n}\right\rangle^{[n][n]}\left\langle i, \Gamma_{i}, \gamma_{i} ; e_{n}\right| .
\end{aligned}
$$

Here $\beta=1 / k_{B} T$ and the partition function is expressed as

$$
\mathcal{Z}=\sum_{n=0}^{N} \sum_{i \in D} \operatorname{dim}(i) e^{-\beta E_{i}^{n}} d^{N-n},
$$

with $d$ the dimension of the Hilbert space at each added site of the Wilson chain, and $d^{N-n}$ the dimension of the space of the "environment" states $e_{n}$. We remark that in the last iteration all states are considered to be discarded, while in the first few iterations there are typically no discarded states yet.

To compute local observables and spectral functions of observables at the impurity site, one traces out step by step the environment states, and introduces the following set of truncated reduced density matrices,

$$
R^{[n]} \equiv \operatorname{Tr}_{\left\{e_{n}\right\}}\left\{\sum_{m \geqslant n} \varrho^{[m]}\right\} .
$$

By symmetry, the reduced density matrices are invariant under the symmetries used, and have therefore a block-diagonal structure in the representation indices. ${ }^{12}$ The matrices $R^{[n-1]}$ can be constructed iteratively from $R^{[n]}$ by tracing out site $n$ and then adding the contribution of states discarded in iteration $(n-1) \rightarrow n$. The contribution of the kept states $(K)$ reads

$$
\left\langle i\left\|R^{[n-1]}\right\| j\right\rangle_{i, j \in K}^{[n-1]}=\widetilde{\sum_{u, v, \mu}} \frac{\operatorname{dim}(u)}{\operatorname{dim}(i)}\left\langle u\left\|R^{[n]}\right\| v\right\rangle^{[n]} .
$$

Here the tilde indicates that the summation runs over states $u$ and $v$ having the same symmetry, and constructed from states $i$ and $j$ by adding the same local state, $i \otimes \mu \rightarrow u$ and $j \otimes \mu \rightarrow v$. The subscript indicates that $i$ and $j$ are both kept states. The discarded piece of $R^{[n-1]}$ is then simply

$$
\left\langle i\left\|R^{[n-1]}\right\| j\right\rangle_{i, j \in D}^{[n-1]}=\delta_{i, j} \frac{d^{N+1-n}}{\mathcal{Z}} e^{-\beta E_{i}^{n-1}} .
$$

To gain insight to the dynamical properties of a quantum impurity, one usually computes the retarded Green's functions for some operator multiplets $A_{\gamma_{A}}$ and $B_{\gamma_{B}}$,

$$
G_{\gamma_{A}, \gamma_{B}}^{\mathrm{ret}}(\omega) \equiv-\frac{i}{\hbar} \int_{0}^{\infty} \operatorname{Tr}\left\{\varrho\left[\left(A_{\gamma_{A}}\right)^{\dagger}(t), B_{\gamma_{B}}(0)\right]_{\xi}\right\} e^{i \omega t} d t
$$

with $\xi=-(\xi=+)$ referring to commutators (anticommutators) appearing for bosonic (fermionic) operators. By 
TABLE I. Irreducible tensor operators for the SU(3) symmetry.

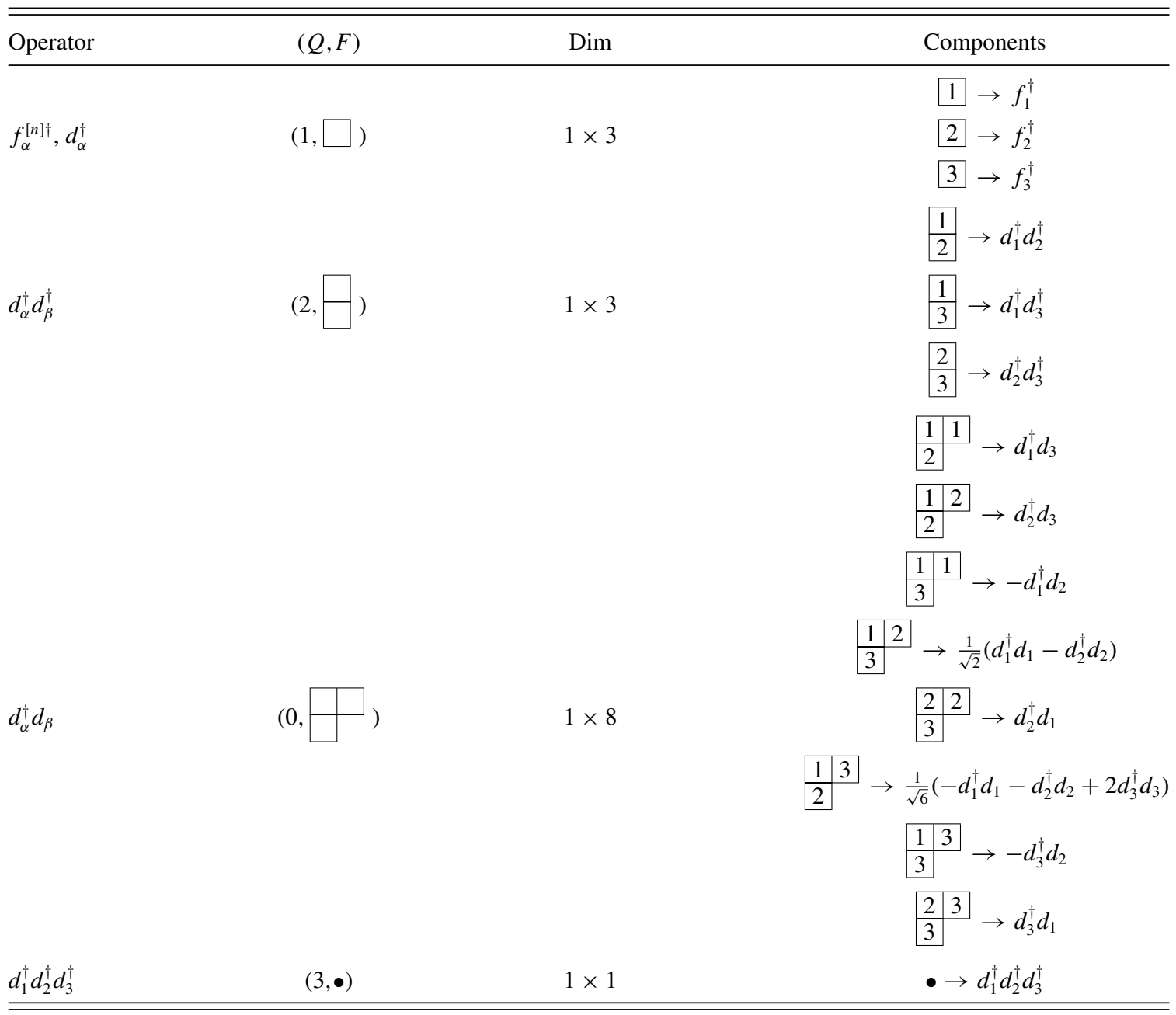

symmetry, to have a nonvanishing value, $A$ and $B$ must both transform according to the same representation, $\Gamma_{A} \cong \Gamma_{B}$, and $\gamma_{A} \equiv \gamma_{B}$ must also be satisfied, $G_{\gamma_{A}, \gamma_{B}}^{\mathrm{ret}}=\delta_{\gamma_{A}, \gamma_{B}} G_{A^{\dagger}, B}^{\mathrm{ret}}$. Notice that Eq. (26) is defined in terms of $\left(\hat{A}_{\gamma_{A}}\right)^{\dagger}$, transforming according to the conjugate representation $\Gamma_{A}^{*}{ }^{*}{ }^{24}$

The expression above can be evaluated in terms of the truncated density matrices, $R^{[n]}$, and the reduced matrix elements $\langle i\|A\| j\rangle_{\alpha}^{[n]}$ and $\langle i\|B\| j\rangle_{\alpha}^{[n]}$ of the operators $A$ and $B$, to obtain ${ }^{12,26}$

$$
\begin{aligned}
G_{A^{\dagger}, B}^{\mathrm{ret}}(z)= & \sum_{n=0}^{N} \sum_{i \in D, K} \sum_{(j, k) \notin(\mathrm{K}, \mathrm{K})}\left\langle i\left\|R^{[n]}\right\| j\right\rangle^{[n]} \\
& \times\left[\sum_{\alpha} \frac{\langle k\|A\| j\rangle_{\alpha}^{[n] *}\langle k\|B\| i\rangle_{\alpha}^{[n]}}{z+\frac{1}{2}\left(E_{i}^{n}+E_{j}^{n}\right)-E_{k}^{n}} \frac{\operatorname{dim}(k)}{\operatorname{dim}(A)}\right. \\
& \left.-\xi \sum_{\alpha} \frac{\langle j\|B\| k\rangle_{\alpha}^{[n]}\langle i\|A\| k\rangle_{\alpha}^{[n] *}}{z-\frac{1}{2}\left(E_{i}^{n}+E_{j}^{n}\right)+E_{k}^{n}} \frac{\operatorname{dim}(i)}{\operatorname{dim}(A)}\right] .
\end{aligned}
$$

This expression provides an efficient way to compute spectral functions. Notice that it contains only the reduced matrix elements and the dimensions of the multiplets involved.

\section{STUDY OF THE SU(3) ANDERSON MODEL}

To demonstrate how the scheme presented above works, we apply it to study the repulsive SU(3)-symmetrical Anderson model, defined already in the introduction. We perform our calculations for a conduction band with a uniform local density of states between energies $W>\epsilon>-W$ with the bandwidth set to $W \equiv 1$, and use the corresponding hopping amplitudes $h^{[n]} \simeq(1 / 2)\left(1+\Lambda^{-1}\right) \Lambda^{-n / 2}$ in Eq. (4). In this case, the width of the (noninteracting) level is approximately given by $\Delta=$ $\pi \varrho_{c} \tilde{V}^{2}$ with $\varrho_{c}=1 / 2 W$ the local density of states at site 0 of the Wilson chain.

As mentioned before, the Hamiltonians (1), (3), and (4) have a $U(1) \times S U(3)$ symmetry in the charge and flavor sectors, respectively. Correspondingly, multiplets of the Hamiltonian are characterized by a charge and a flavor quantum number. The charge quantum numbers $Q_{i}$ are simply identical to the total charge, ${ }^{28}$

$$
Q \equiv \sum_{\alpha}\left\{\sum_{n=0}^{N}\left(f_{\alpha}^{[n] \dagger} f_{\alpha}^{[n]}-\frac{3}{2}\right)+\left(d_{\alpha}^{\dagger} d_{\alpha}-\frac{3}{2}\right)\right\},
$$

conserved by Eqs. (1), (3), and (4).

Labeling the SU(3) representations and the states within an SU(3) multiplet is somewhat more complicated. The flavor 
TABLE II. Energy spectrum for the electron-hole symmetric, mixed valence point. $E_{L}$ stands for the finite size energy scale, $E_{L}=2 \pi / L=1.311 . Q_{0}=0(3 / 2)$ for even (odd) iterations.

\begin{tabular}{|c|c|c|c|c|c|}
\hline & $E /(2 \pi / L)$ & $E_{\mathrm{NRG}} / E_{L}$ & $Q$ (charge) & $\mathrm{SU}(3)$ & Dimension \\
\hline \multirow{8}{*}{ Even iterations } & 0 & 0 & $Q_{0}$ & $\bullet$ & 1 \\
\hline & $1 / 2$ & 0.5 & $Q_{0}+1$ & $\square$ & 3 \\
\hline & $1 / 2$ & 0.5 & $Q_{0}-1$ & & 3 \\
\hline & 1 & 1 & $Q_{0}+2$ & & 3 \\
\hline & 1 & 1 & $Q_{0}$ & & 8 \\
\hline & 1 & 1 & $Q_{0}$ & $\bullet$ & 1 \\
\hline & 1 & 1 & $Q_{0}-2$ & $\square$ & 3 \\
\hline & $E /(2 \pi / L)$ & $E_{\mathrm{NRG}} / E_{L}$ & $Q$ (charge) & $\mathrm{SU}(3)$ & Dimension \\
\hline \multirow{12}{*}{ Odd iterations } & 0 & 0 & $Q_{0}$ & $\bullet$ & 1 \\
\hline & 0 & 0 & $Q_{0}-1$ & & 3 \\
\hline & 0 & 0 & $Q_{0}-2$ & $\square$ & 3 \\
\hline & 0 & 0 & $Q_{0}-3$ & $\bullet$ & 3 \\
\hline & 1 & 0.98 & $Q_{0}+1$ & & 3 \\
\hline & 1 & 0.98 & $Q_{0}$ & & 8 \\
\hline & 1 & 0.98 & $Q_{0}$ & $\bullet$ & 1 \\
\hline & 1 & 0.98 & $Q_{0}-1$ & 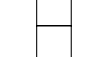 & 3 \\
\hline & 1 & 0.98 & $Q_{0}-1$ & & 6 \\
\hline & 1 & 0.98 & $Q_{0}-1$ & \begin{tabular}{|l} 
\\
\end{tabular} & 3 \\
\hline & 1 & 0.98 & $Q_{0}+1$ & 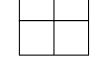 & 6 \\
\hline & 1 & 0.98 & $Q_{0}+2$ & $\square$ & 3 \\
\hline
\end{tabular}

quantum numbers $F_{i}$ can be (and are usually) represented by Young tableaux, characterized by two non-negative integers in case of SU(3) (see Appendix A). Young tableaux provide a nice pictorial way to multiply and decompose representations, or calculate their dimensions. For our numerical calculations, however, we had to construct explicitly the basis states of SU(3) representations and to compute the corresponding Clebsch-Gordan coefficients. ${ }^{29}$ This we carried out using the so-called Gelfand-Tsetlin patterns, briefly discussed in Appendix A. The states obtained this way are analogs of the canonical SU(2) basis states, created by the raising and lowering spin operators $S^{ \pm}$. Gelfand-Tsetlin patterns are in one-to-one correspondence with the Young tableaux, but they allow for a simpler explicit construction of the basis states. For more details, we refer the reader to Ref. 16.

Similar to the eigenstates and multiplets, we also need to group operators into SU(3) multiplets and characterize them by appropriate $\mathrm{SU}(3)$ quantum numbers. This classification of the most important operators is summarized in Table I. In terms of $\mathrm{SU}(3)$, there is only a single hopping operator,
$C_{\gamma}^{[n]} \leftrightarrow f_{\alpha}^{[n] \dagger}$, which transforms according to the defining SU(3) representation and has charge $Q=1$, similar to the creation operator of the localized level $d_{\alpha}^{\dagger}$. From $d_{\alpha}^{\dagger}$ we can also construct various local operators of interest. The spin operators, $\sim d_{\alpha}^{\dagger} d_{\beta}$ form, for example, an eight-dimensional charge $Q=0$ operator multiplet in terms of $\mathrm{U}(1) \times \mathrm{SU}(3)$, while the charge $Q=2$ pairing operators $\sim d_{\alpha}^{\dagger} d_{\beta}^{\dagger}$ transform according to a three-dimensional SU(3) representation. Finally, the "trion" operator $d_{1}^{\dagger} d_{2}^{\dagger} d_{3}^{\dagger}$ has charge $Q=3$ and is an SU(3) singlet.

\section{A. Numerical results}

In our runs we have kept about 250 multiplets at each iteration, corresponding to approximately 1500 states on average, while the Wilson parameter was fixed to $\Lambda=2$.

In Fig. 1 we present the occupation of the localized level as a function of the energy $\varepsilon_{d}$. The localized level can accommodate up to three fermions. For large enough $U / \Delta$ 's, these fermions enter the local level one by one as $\varepsilon_{d}$ is decreased, and a Coulomb staircase is clearly visible. In 


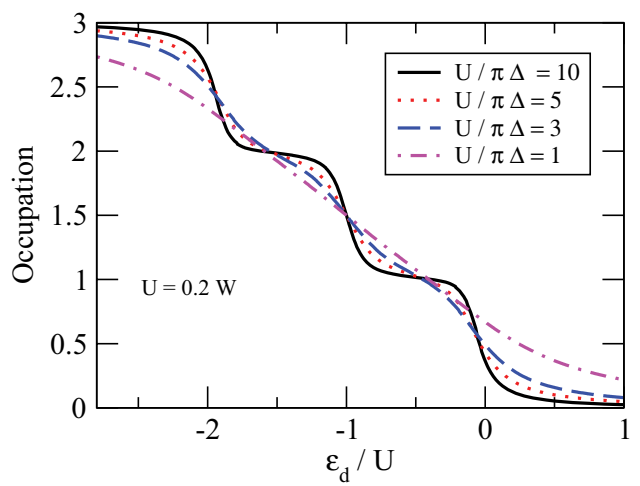

FIG. 1. (Color online) The occupation number as a function of $\varepsilon_{d}$ and for different broadening parameters $\Delta$. The temperature is $T=0$ and the Coulomb interaction is fixed to $U=0.2 \mathrm{~W}$.

the range $-U<\varepsilon_{d}<0$ there is approximately one electron on the level. The SU(3) spin of this electron is screened by the conduction electrons below the Kondo scale $T_{K}$, and an $\mathrm{SU}(3)$ Kondo state is formed. A similar, holelike $\mathrm{SU}(3)^{*}$ state emerges for $-2 U<\varepsilon_{d}<-U$.

The $\mathrm{SU}(3)\left(-U<\varepsilon_{d}<0\right)$ Kondo temperature can be estimated by first doing perturbation theory in $\tilde{V}$ and performing a Schrieffer-Wolff transformation and then carrying out a renormalization group analysis. This analysis yields a Kondo temperature of

$$
T_{K}^{\mathrm{SU}(3)} \approx D_{0} e^{-1 / 3 \lambda},
$$

with the dimensionless coupling $\lambda$ expressed as

$$
\lambda=\frac{\Delta}{\pi E_{+}}+\frac{\Delta}{\pi E_{-}},
$$

in terms of the level width $\Delta$ and the "ionization energies" $E_{+}=U+\epsilon_{d}$ and $E_{-}=-\epsilon_{d}$, and $D_{0} \approx \min \left(E_{+}, E_{-}\right)$a high energy cutoff.

The most intriguing region is, however, the mixed valence region, $\varepsilon_{d} \approx-U$. For $\varepsilon_{d}=-U$ the ground state of the isolated
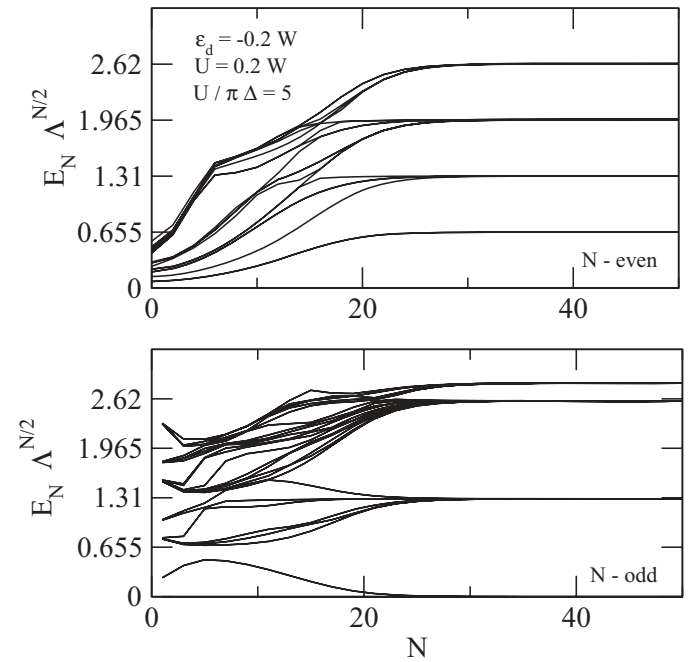

FIG. 2. NRG finite size spectrum at half filling and $T=0$. Upper (lower) panel represents the even (odd) part of the spectrum. In both plots we represent the lowest 50 energy levels. The parameters are as follows: $\Lambda=2, U=0.2 \mathrm{~W}, \varepsilon_{d}=-U$, and $U / \pi \Delta=5$. The convergence was reached after approximately 27 iterations.

impurity $(\Delta=0)$ would be sixfold degenerate due to electronhole symmetry, connecting the two SU(3) triplets, $\left\{d_{\alpha}^{\dagger}|0\rangle\right\}$ and $\left\{d_{\alpha}^{\dagger} d_{\beta}^{\dagger}|0\rangle\right\}$. Valence fluctuations produce a state with all these states strongly mixed by quantum fluctuations. Figure 2 and Table II show the flow diagram of the NRG levels, and the $\mathrm{SU}(3)$ classification of the asymptotic finite size spectrum, respectively. A detailed analysis reveals that this finite size spectrum can simply be understood as the finite size spectrum of three chiral fermions with a phase shift $\delta=\pi / 2$ at the Fermi energy. This phase shift is indeed in full agreement with the Friedel sum rule, $3 \delta / \pi=\langle n\rangle$, and the occupation $\langle n\rangle=3 / 2$ assured by electron-hole symmetry.

Similarly, the SU(3) Kondo spectrum, displayed in Table III can be understood as the finite size spectrum of three chiral

\begin{tabular}{|c|c|c|c|c|c|}
\hline & $E /(2 \pi / L)$ & $E_{\mathrm{NRG}} / E_{L}$ & $Q$ (charge) & $\mathrm{SU}(3)$ & Dimension \\
\hline \multirow{7}{*}{ Even iterations } & 0 & 0 & $Q_{0}$ & $\bullet$ & 1 \\
\hline & $2 / 3$ & 0.33 & $Q_{0}-1$ & $\mid$ & 3 \\
\hline & $4 / 3$ & 0.66 & $Q_{0}+1$ & $\square$ & 3 \\
\hline & $4 / 3$ & 0.66 & $Q_{0}-2$ & $\square$ & 3 \\
\hline & 2 & 1 & $Q_{0}$ & \begin{tabular}{|l|l} 
\\
\end{tabular} & 8 \\
\hline & 2 & 1 & $Q_{0}$ & • & 1 \\
\hline & 2 & 1 & $Q_{0}-3$ & $\bullet$ & 1 \\
\hline \multirow{4}{*}{ Odd iterations } & $E /(2 \pi / L)$ & $E_{\mathrm{NRG}} / E_{L}$ & $Q$ (charge) & $\mathrm{SU}(3)$ & Dimension \\
\hline & 0 & 0 & $Q_{0}$ & $\bullet$ & 1 \\
\hline & $1 / 3$ & 0.15 & $Q_{0}+1$ & $\square$ & 3 \\
\hline & $2 / 3$ & 0.32 & $Q_{0}+2$ & 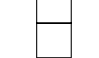 & 3 \\
\hline
\end{tabular}

TABLE III. Energy spectrum in the Kondo regime. $Q_{0}=0(-3 / 2)$ for even (odd) iterations. 


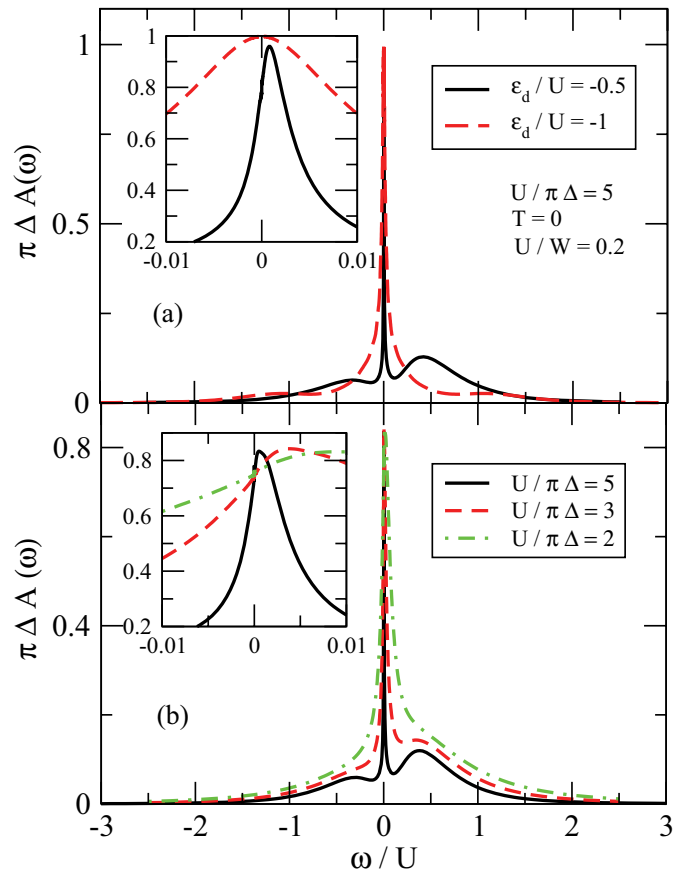

FIG. 3. (Color online) (a) The normalized spectral function for the on-site creation operator $d_{\alpha}^{\dagger}$ for two different fillings $n=1.5$ (half filling, red dashed) and $n=1$ (1/3 filling, black solid). In the singly occupied case, $n=1$, the dot is in the Kondo regime, and the resonance is shifted away from the Fermi energy, $\omega=0$. The value of the spectral function at the Fermi energy is determined by the Friedel sum rule, $\pi \Delta A(0) \rightarrow 3 / 4$. (b) Evolution of the normalized spectral function as a function of $U / \pi \Delta$ in the Kondo regime, for $\varepsilon_{d} / U=$ -0.5 . The deviation from the Friedel sum rule is less than $1 \%$.

fermions with a phase shift $\delta \approx \pi / 3$, implied by the Friedel sum rule and the occupation $\langle n\rangle \approx 1$.

\section{B. Spectral functions of local operators}

The crossover between the two SU(3) Kondo regimes through the mixed valence regime is maybe best captured by the local level's spectral function, shown in Fig. 3. In the SU(3) and $\mathrm{SU}(3)^{*}$ Kondo regimes we find a narrow Kondo resonance of an exponentially small width pinned somewhat asymmetrically to the Fermi energy, $\omega=0$ (see inset), and two Hubbard peaks. At the mixed valence point, $\varepsilon_{d}=-U$ on the other hand, a relatively broad and symmetrical resonance of width $\sim \Delta$ appears at the Fermi energy, and the charging peaks are absent.

The behavior of strongly correlated cold atomic and heavy fermion lattice systems can often be understood in terms of a self-consistent quantum impurity model (dynamical meanfield theory). Within this picture, the local response functions of the quantum impurities may drive superconducting or magnetic phase transitions, or lead to even more exotic quantum phases. In this subsection, let us therefore analyze the spectral properties of the SU(3) Anderson model, and investigate the local spectral and response functions of its spin, pairing, and trion operators.

As discussed before, the spin operators $d_{\alpha}^{\dagger} d_{\beta}$ transform according to an eight-dimensional SU(3) representation. As shown in Fig. 4, their spectral function displays Fermi liquid properties (see inset), and behaves very similarly to the spin

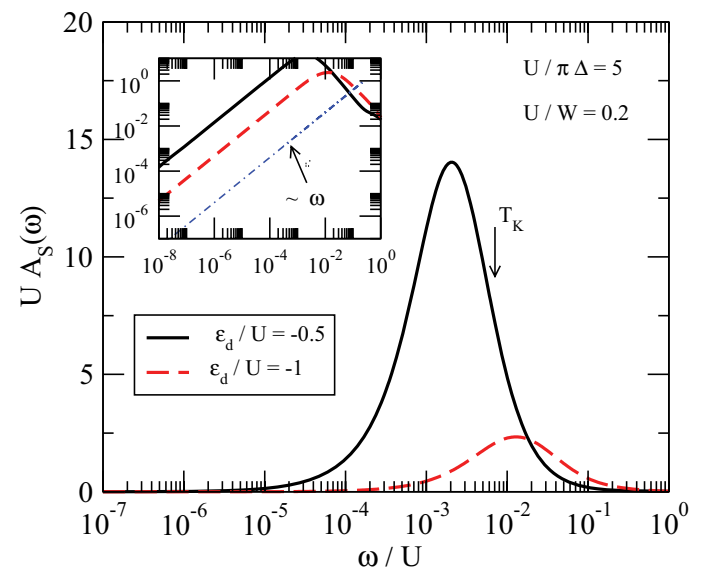

(a)

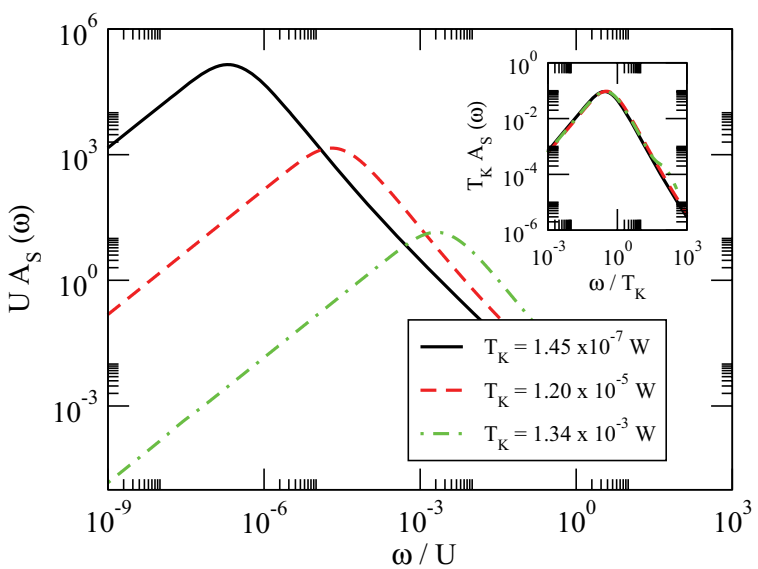

(b)

FIG. 4. (Color online) (Upper panel) Spectral function of the spin operator $d_{\alpha}^{\dagger} d_{\beta}$ on a logarithmic scale for two different fillings, $\langle n\rangle=1.5$ (half filling, red dashed) and $\langle n\rangle \approx 1$ (1/3 filling, black solid) for $U=0.2 \mathrm{~W}$. In the singly occupied case, $\langle n\rangle \approx 1$, the dot is in the Kondo regime. The inset indicates the linear decay in $A_{S}(\omega)$ in the small frequency limit. The blue, dashed-dotted line is a guideline for the eye. (Lower panel) The spectral function of the spin operator in the Kondo regime, $\langle n\rangle \approx 1$, and for three different ratios $U / \pi \Delta$ : $U / \pi \Delta=15$ (solid black line), $U / \pi \Delta=10$ (dashed red line), and $U / \pi \Delta=5$ (dashed-dotted green line). The inset indicates the universal scaling collapse of the spin spectral function in the Kondo regime.

spectral function of a standard SU(2) Anderson model. ${ }^{30}$ In the mixed valence regime, for $\Delta \ll U$ charge fluctuations to the state $n=0$ and $n=3$ are frozen out, and at low energies the only relevant energy scale is $\Delta$; correspondingly, the spectral function exhibits a broad resonance at $\omega \sim \Delta$ (extending up to $\omega \sim U$ ), and decays linearly to zero for small frequencies, $A_{S}^{n \approx 3 / 2}(\omega) \sim \omega / \Delta^{2}$. By Hilbert transform, this amounts in a spin susceptibility, $\chi_{S} \sim 1 / \Delta$. In the Kondo regime, $\langle n\rangle \approx 1$, on the other hand, two separate scales can be distinguished. Below $\omega \sim \min \left(U,\left|\epsilon_{d}\right|\right)$ charge fluctuations are frozen and a clear Kondo anomaly can be observed as a logarithmic increase of the spectral function, as indicated by the arrow in Fig. 4. The Fermi liquid behavior only emerges below the Kondo scale, $\omega<T_{K} \ll \Delta, U$. In this Kondo regime $A_{S}^{\text {Kondo }}(\omega) \sim \omega / T_{K}^{2}$, and correspondingly, a susceptibility $\chi_{S} \sim 1 / T_{K}$ is found. 


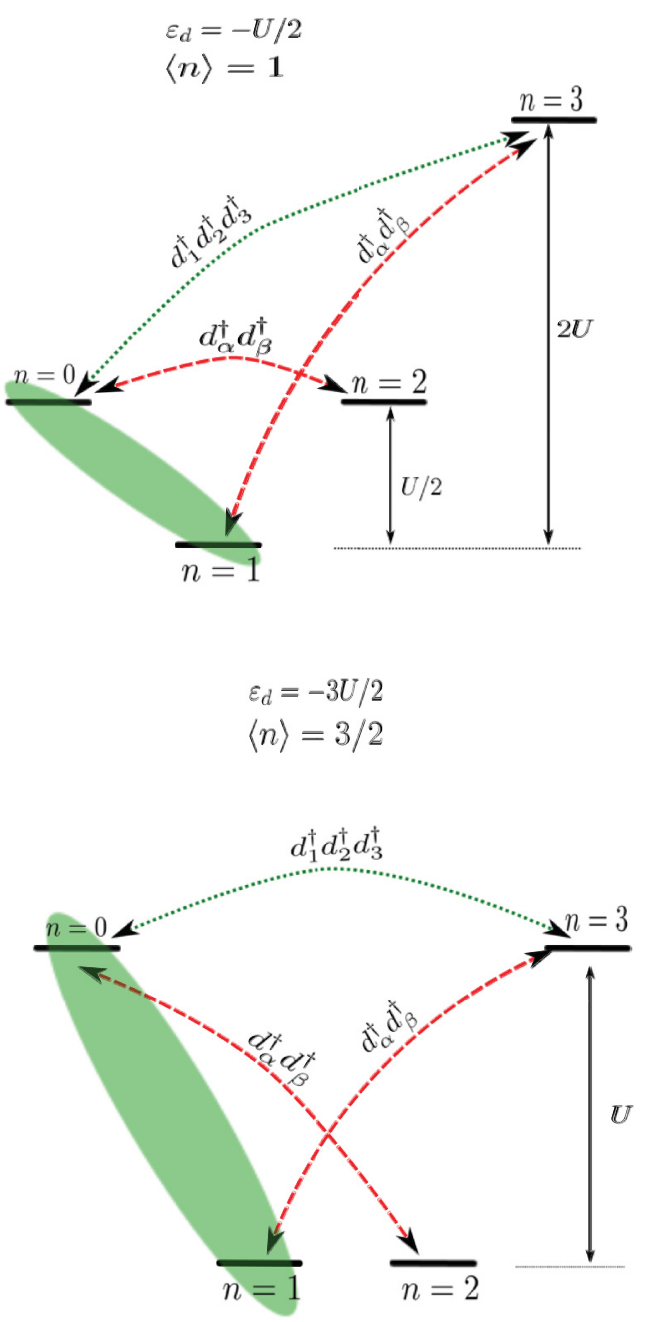

FIG. 5. (Color online) Impurity states for the SU(3) Anderson model in the Kondo (upper panel) and in the mixed valence regime (lower panel). The green shaded area emphasizes that quantum fluctuations mix the $n=1$ and $n=0$ levels. The arrows indicate possible direct transitions that are responsible for the peaks observed in the spectral functions of the superconducting $\left(d_{\alpha}^{\dagger} d_{\beta}^{\dagger}\right)$ and trion $\left(d_{1}^{\dagger} d_{2}^{\dagger} d_{3}^{\dagger}\right)$ operators.

The spin spectral function becomes universal in the Kondo limit, $T_{K} \ll \Delta, U$ in the sense that the $T=0$ temperature dynamical susceptibility scales as $\chi_{S}(\omega)=$ $\left(1 / T_{K}\right) f\left(\omega / T_{K},\langle n\rangle\right)$, with $f\left(\omega / T_{K},\langle n\rangle\right)$ a function, which only slightly depends on the occupation of the level, $\langle n\rangle \approx$ 1. This is demonstrated in the lower panel of Fig. 4 for the imaginary part of the susceptibility, $\chi_{S}^{\prime \prime}(\omega)=-\pi A_{S}(\omega)$, computed for different values of $U / \pi \Delta$. Numerically we define $T_{K}$ as the half width at half maximum of $A(\omega)$, the spectral function of the $d$ operator of the localized level.

The correlations of the pairing operators $d_{\alpha}^{\dagger} d_{\beta}^{\dagger}$ behave somewhat similar to those of the spin in the sense that at small frequencies a linear frequency dependence is found, corresponding to a Fermi liquid state with a constant pairings susceptibility (see inset of Fig. 6). To understand the spectrum at large frequencies, $\omega \sim U$, we present in Fig. 5 the impurity states for the decoupled Hamiltonian. In the Kondo regime, the lowest lying impurity state is the $n=1 \mathrm{SU}(3)$ triplet. The

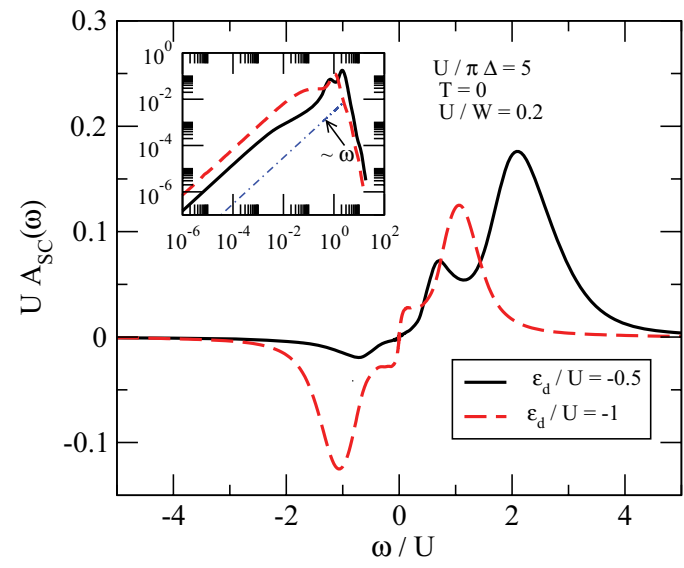

FIG. 6. (Color online) Spectral function of the superconducting operator for $U=0.2 D$ and $\langle n\rangle=1.5$ (half filling, red dashed) and $\langle n\rangle \approx 1$ (1/3 filling, black solid).

corresponding excitation spectrum is shown for $\varepsilon_{d}=-U / 2$ in Fig. 5. In the superconducting spectral function several peaks appear which can be assigned to transitions between impurity states. The maximum weight is expected to be on the particle-side of the spectrum at $\omega \sim 2 U$ ( $n=1 \rightarrow 3$ transition). A peak of smaller amplitude emerges at $\omega \sim U / 2$. This peak is due to quantum fluctuations, which mix the $n=1$ and $n=0$ states, and thus allow for a direct transition between the $n=1$ and $n=2$ states. Similarly, a holelike excitation appears at $\omega \sim-U / 2$, which, in turn can be explained by quantum fluctuations mixing the lowest lying $n=1$ and the $n=2$ states. In the mixed valence regime the $n=1$ and $n=2 \mathrm{SU}(3)$ triplets form the lowest lying impurity states. There transitions between these states and the $n=0$ and $n=2$ excited states of energy $\Delta E \approx U$ is only possible due to quantum fluctuations. Correspondingly, two relatively large peaks appear at $\omega \sim \pm U$ in the superconducting spectral function.

As a final example, we display the spectral function of the SU(3) singlet trion operator, $T^{\dagger}=d_{1}^{\dagger} d_{2}^{\dagger} d_{3}^{\dagger}$ in Fig. 7. The trion operator plays an important role in the attractive case, ${ }^{31}$ however, in this repulsive model it is a highly suppressed operator. In the Kondo regimes, it has a nonzero spectral function only

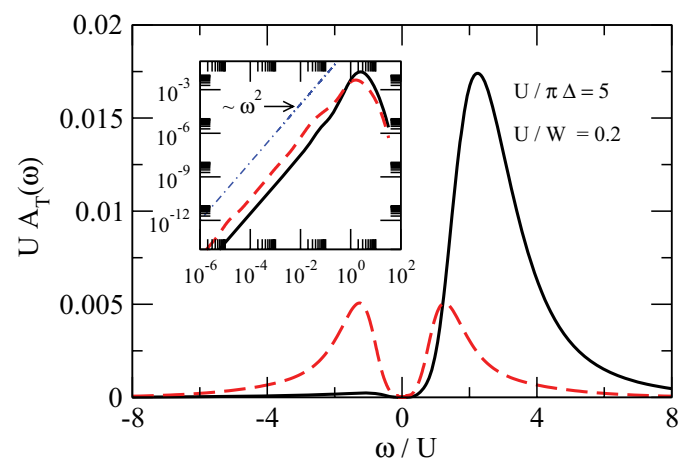

FIG. 7. (Color online) The spectral function for the trion operator for two different fillings $n=1.5$ (half filling, red dashed) and $n=1$ (1/3 filling, black solid). The inset represents the same data on a logarithmic scale. The trionic spectral function scales as $\omega^{2}$ in the small energy limit. 


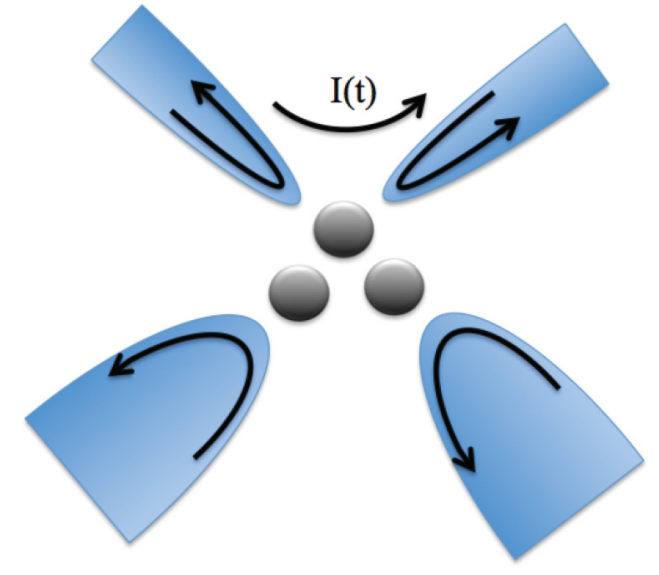

FIG. 8. (Color online) Possible realization of SU(3) Kondo states using four edge states and three quantum dots. The upper edge state is splitted and allows transport measurements between the two upper external leads. ${ }^{9}$

because the Kondo states have a small $(\sim \Delta / U)$ admixture of the empty and the triply occupied states, respectively (see also Fig. 5). This explains why the amplitude of the signal is in this case smaller in the mixed valence regime. It also explains the strong electron-hole asymmetry in the Kondo regimes. In the $\langle n\rangle \approx 1$ regime, shown in Fig. 7, for example, the admixture of the $n=0$ state is relatively large, $\sim \Delta / U$, while the $n=3$ state has a much smaller weight, $\sim(\Delta / U)^{2}$. As a consequence, most of the spectral weight appears on the particlelike side of the spectral function, $\omega \approx 2 U$ (see Fig. 5). At small frequencies the spectral function decays as $\sim \omega^{2}$. This is in agreement with Fermi liquid theory, which would predict $\mathrm{a}\left\langle T(t) T^{\dagger}(0)\right\rangle \sim 1 / t^{3}$ decay of the trionic correlation functions at very long times.

\section{Conductance}

Let us close this section by discussing the finite temperature conductance through a mesoscopic system that was proposed to support the $\mathrm{SU}(3)$ Kondo state. ${ }^{9}$ A sketch of the device is shown in Fig. 8. It consists of three capacitively interacting quantum dots, tuned to a state, where the three charging states of the dots, $\left(n_{1}, n_{2}, n_{3}\right)=(1,0,0),(0,1,0)$, and $(0,0,1)$ are degenerate. The spin degeneracy of the dots is then removed by a large external magnetic field, and the three dots are tunnel coupled to leads supporting chiral quantum-Hall edge states. In the limit, where there is no tunneling between the dots, this system supports a charge SU(3) Kondo state, where the localized electron in the dots is screened by the conduction electrons in the external leads. ${ }^{9}$ We remark that this degeneracy point has, in fact, been reached with a somewhat different three-dot device studied in Ref. 32, but there no conductance measurement has been performed and the leads were not in the quantum Hall regime either.

To measure the conductance through the device, one needs to attach two electrodes to at least one of the dots, as shown in Fig. 8. In this configuration, the conductance can be directly related to the spectral functions of the $d$ level, and for a symmetrical device one finds

$$
G(T)=\pi \Delta \frac{e^{2}}{h} \int_{-\infty}^{\infty} d \omega A(\omega, T)\left(-\frac{\partial f(\omega)}{\partial \omega}\right) .
$$

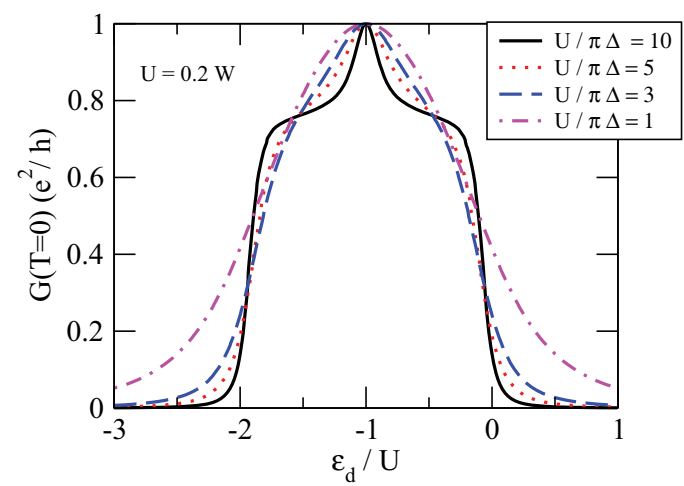

FIG. 9. (Color online) The conductance as a function of $\varepsilon_{d}$ and for different broadening parameters $\Delta$. The temperature is $T=0$ and the Coulomb interaction is fixed to $U=0.2 \mathrm{~W}$.

Here $A(\omega)$ is the spectral function of the $d_{\alpha}$ operator of the localized level, $A(\omega)=-\Im m G_{d_{\alpha} d_{\beta}^{\dagger}}(\omega) / \pi$, with $G_{d_{\alpha} d_{\beta}^{\dagger}}(\omega)$ the Green's function defined in Eq. (26). The corresponding $T=0$ temperature linear conductance is shown in Fig. 9 as a function of the energy $\epsilon_{d}$. The conductance reaches its maximal value in the mixed valence regime, $\varepsilon_{d} \sim-U$, and displays $\mathrm{SU}(3)$ Kondo effect related plateaus in the regions $-2 U<\varepsilon_{d}<-U$ and $-U<\varepsilon_{d}<0$. The $T=0$ temperature conductance values observed can be understood in terms of the Friedel sum rule, relating the total occupation of the $d$ level to the phase shift of the conduction electrons, which yields $3 \delta / \pi=\langle n\rangle$ for the $\mathrm{SU}(3)$ Anderson model. ${ }^{9}$ In a Fermi liquid state-at $T=0$ temperature-the conductance can be computed using the Landauer-Büttiker formula, and in the geometry of Fig. 8 is simply related to the phase shift as $G(T=0)=\left(e^{2} / h\right) \sin ^{2}(\delta)$. This explains the value $G_{\mathrm{SU}(3)} \approx(3 / 4) e^{2} / h$ observed in the Kondo states; there the occupancies are $\langle n\rangle \approx 1$ and $\langle n\rangle \approx 2$, corresponding to phase shifts $\delta= \pm \pi / 3$, and the previously mentioned value of the conductance. At the mixed valence point, $\varepsilon_{d}=-U$, on the other hand, we have $\langle n\rangle=3 / 2$, implying a phase shift $\delta=\pi / 2$ and a maximal conductance $G_{\mathrm{SU}(3)}=e^{2} / h$.

Increasing the temperature, the conductance is quickly suppressed in the Kondo regimes, and three Coulomb blockade conductance peaks emerge at the points of charge degeneracy, as shown in Fig. 10. The central peak corresponds to the transition $n=1 \leftrightarrow 2$ while the two side peaks correspond to charge fluctuations $n=1 \leftrightarrow 0$ and $n=2 \leftrightarrow 3$, respectively. In Fig. 10 we also display the temperature dependence of the conductance at the mixed valence point and in the SU(3) Kondo regime. The Kondo temperature of the SU(3) Kondo state is clearly much smaller than the mixed valence energy scale even for these moderate interactions.

\section{CONCLUSIONS}

In this paper, we showed how to extend the DM-NRG scheme of Ref. 12 to symmetries with outer multiplicities. As an application, we performed a detailed DM-NRG study of the SU(3) symmetrical Anderson model by first incorporating $\mathrm{SU}(N)$ symmetries $^{16}$ in the Open Access Budapest DM-NRG code ${ }^{13}$ and then performing the numerical calculations using the complete $\mathrm{U}(1) \times \mathrm{SU}(3)$ symmetry of the model. A similar 


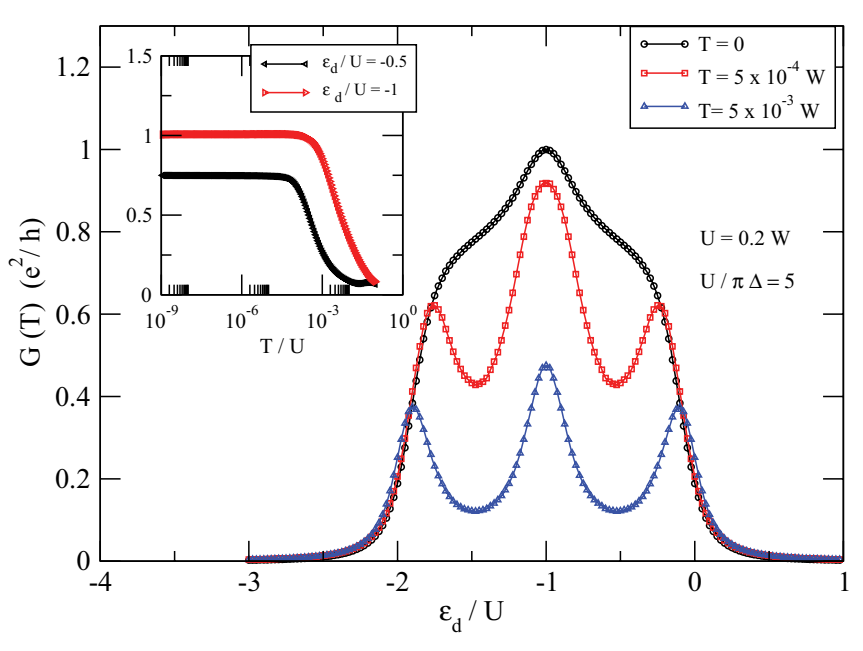

FIG. 10. (Color online) Finite temperature conductance as a function of $\varepsilon_{d}$. (Inset) The temperature dependence of the conductance on a logarithmic scale, for two different fillings, $n=1.5$ (half filling, red dashed) and $n=1$ (1/3 filling, black solid). In both panels the Coulomb energy and the broadening to the leads were fixed to $U=0.2 W$ and $U / \pi \Delta=5$.

extension has been carried out within the matrix product state (MPS) approach parallel to this work. ${ }^{15}$

The properties of the SU(3) Anderson model do not differ so much from those of the original Anderson model. As also discussed in Ref. 9, for $U>\Delta$ four distinct charging regions appear: the featureless empty and fully occupied regions $(\langle n\rangle \approx 3$ and $\langle n\rangle \approx 0)$, and two Kondo regions of occupancies $\langle n\rangle \approx 1$ and $\langle n\rangle \approx 2$, respectively. The two $\mathrm{SU}(3)$ Kondo regions behave similarly: They are characterized by phase shifts $\delta \sim \pm \pi / 3$, as verified from the finite size spectrum, and correspondingly, a Kondo resonance shifted away from the Fermi energy. In these Kondo regimes, the susceptibility has a universal form, $\chi(\omega)=f\left(\omega / T_{K},\langle n\rangle\right) / T_{K}$, with a scaling function $f(x,\langle n\rangle)$ very similar to the one emerging in the SU(2) Anderson and Kondo models. For completeness, we also studied the spectral properties of other local operators such as pairing or the trion operators. Both of them turn out to have a small amplitude for $\Delta \ll U$, and exhibit simple Fermi liquid properties below the mixed valence and Kondo scales, respectively. Therefore, away from half filling, a magnetic instability is expected to prevail on a lattice in the SU(3) Hubbard model, in general agreement with the results of Gutzwiller calculations at low temperatures. ${ }^{27}$

The SU(3) Kondo regions are separated by a mixed valence state, which again has a Fermi liquid character with a Fermi liquid scale of the order of the level width, $\Gamma$. Here we find a phase shift $\delta=\pi / 2$, in agreement with the expectations based upon the Friedel sum rule, but apart from that, and the emerging electron-hole symmetry at this point, the properties of the mixed valence state appear to be very quite similar to those of the Kondo states.

We also investigated the conductance properties of the SU(3) arrangement, proposed in Ref. 9. At high temperatures we observe in the side conductance three charging peaks, corresponding to the three charging steps. As the temperature is lowered, the Coulomb blockade valleys are gradually filled up, and two conductance shoulders emerge in the Kondo regime with a conductance $G \approx(3 / 4)\left(e^{2} / h\right)$.

The methods and the computations presented here represent a first and important step to perform DM-NRG and DMFT calculations for more elaborate $\mathrm{SU}(N)$ or $\mathrm{Sp}(N)$ lattice models. However, further work is necessary to optimize these DMFT calculations. While we definitely gain enormous storage space by using $\mathrm{SU}(N)$ symmetries, the evaluation of the reduced matrix elements and multiple sums over internal representation labels are currently not carried out with maximal efficiency. Since for large $N$ 's the dimensions of irreducible $\mathrm{SU}(N)$ representations grow very fast, these summations quickly become the bottleneck for DM-NRG calculations, and further work is needed to increase the efficiency of the corresponding subroutines.

\section{ACKNOWLEDGMENTS}

We acknowledge numerous fruitful discussions with A. Weichselbaum. We thank the National Institute for Theoretical Physics in Stellenbosch, South Africa, where this collaboration was started, for hospitality, and the DFG (Grant No. De730/7-1) for financially supporting our visit there. This research has been supported by Hungarian research funds OTKA and NKTH under Grants No. K73361 and No. CNK80991, and the EU-NKTH GEOMDISS project. J.v.D. and A.A. acknowledge financial support from SFB-TR12. C.P.M. has benefit from financial support from UEFISCDI under French-Romanian Grant DYMESYS (ANR 2011-IS04001-01 and Contract No. PN-II-ID-JRP-2011-1), and PN-IIID-PCE-2012-4-0039).

\section{APPENDIX A: SOME DETAILS ON SU $(N)$ REPRESENTATIONS}

In this Appendix we give a brief overview of the representation theory of $\mathrm{SU}(N)$, following the approach of Ref. 16, where a more detailed discussion can be found.

While $\mathrm{SU}(2)$ has three generators, $\hat{\mathcal{J}}_{z}, \hat{\mathcal{J}}_{+}$, and $\hat{\mathcal{J}}_{-}, \mathrm{SU}(N)$ has $N^{2}-1$ generators. We shall deal explicitly with only $3(N-1)$ of them, denoted by $\hat{\mathcal{J}}_{z}^{(l)}$ and $\hat{\mathcal{J}}_{ \pm}^{(l)}$, where $l=$ $1, \ldots, N-1$. By definition, they satisfy the commutation relations,

$$
\left[\hat{\mathcal{J}}_{z}^{(l)}, \hat{\mathcal{J}}_{ \pm}^{(l)}\right]= \pm \hat{\mathcal{J}}_{ \pm}^{(l)}, \quad\left[\hat{\mathcal{J}}_{+}^{(l)}, \hat{\mathcal{J}}_{-}^{(l)}\right]=2 \hat{\mathcal{J}}_{z}^{(l)}
$$

These have the same form as those of the corresponding $N \times$ $N$ matrices,

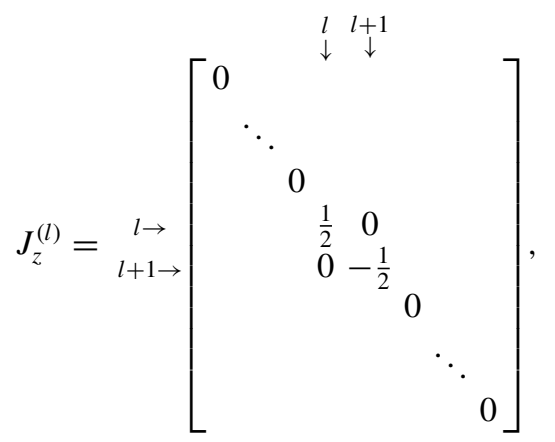




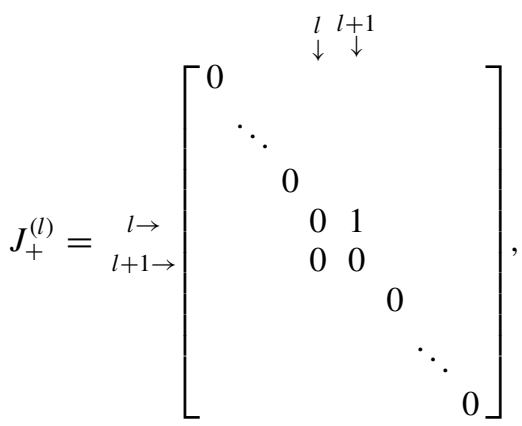

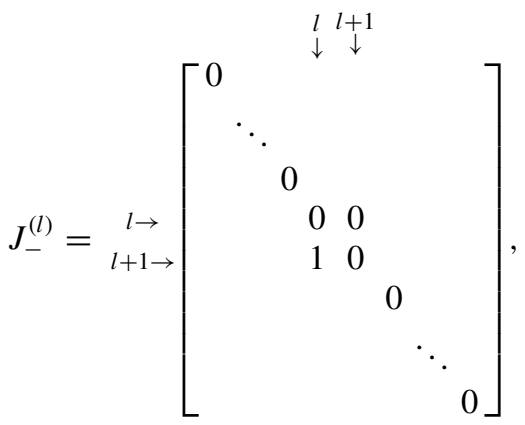

which generate the defining representation of $\mathrm{SU}(N)$. The $N^{2}-3 N+2$ remaining generators are obtained as commutators between generators involving different values of $l$. Below we will use $\hat{\mathcal{J}}^{a}$ (and $J^{a}$ ) as collective notation for any of the generators (and corresponding matrices in the defining representation).

Let $|\Gamma, \gamma\rangle$ denote the basis states of a general $\mathrm{SU}(N)$ irrep, where $\Gamma$ labels the irrep and $\gamma$ its individual basis states. The action of any generator on a basis state can be written as

$$
\hat{\mathcal{J}}^{a}|\Gamma, \gamma\rangle=\sum_{\gamma^{\prime}} \Gamma_{\gamma^{\prime}, \gamma}^{a}\left|\Gamma, \gamma^{\prime}\right\rangle,
$$

where $\Gamma_{\gamma^{\prime}, \gamma}^{a} \equiv\left\langle\Gamma, \gamma^{\prime}\left|\hat{\mathcal{J}}^{a}\right| \Gamma, \gamma\right\rangle$ are the matrix elements of $\hat{\mathcal{J}}^{a}$ within the irrep $\Gamma$. For example, the matrix elements of $\hat{\mathcal{J}}_{z, \pm}^{(l)}$ in the defining representation are given by Eq. (A2).

It is convenient to choose the states $|\Gamma, \gamma\rangle$ to be simultaneous eigenstates of $\hat{\mathcal{J}}_{z}^{(l)}$ for $l=1, \ldots, N-1$, with eigenvalues $\lambda_{l}^{\Gamma, \gamma}$, say. The sequence $W_{z}(\Gamma, \gamma)=\left(\lambda_{1}^{\Gamma, \gamma}, \ldots, \lambda_{N-1}^{\Gamma, \gamma}\right)$ is called its weight. As for $\mathrm{SU}(2)$, all components of the weight take half-integer values.

A convenient way to visualize all states of the same $\mathrm{SU}(N)$ irrep is then given by weight diagrams (see Fig. 11 for an example), which are constructed by marking the point with coordinates $W_{z}(\Gamma, \gamma)$ for each state $|\Gamma, \gamma\rangle$ of an irrep $\Gamma$. The operators $\hat{J}_{ \pm}^{(l)}$ then map states onto their neighbors in a weight $\operatorname{diagram}\left(\lambda_{l}^{\Gamma, \gamma} \rightarrow \lambda_{l}^{\Gamma, \gamma} \pm 1\right)$.

Each irrep $\Gamma$ has a so-called highest-weight state (unique up to a phase), denoted by $|\Gamma, \gamma=\Gamma\rangle$ for convenience. It is annihilated by all $\hat{\mathcal{J}}_{+}^{(l)}$,

$$
\hat{\mathcal{J}}_{+}^{(l)}|\Gamma, \gamma=\Gamma\rangle=0 \quad(l=1, \ldots, N-1) .
$$

Its weight actually determines the properties of the whole irrep $\Gamma$, and is thus suitable to provide a labeling scheme for $\Gamma$.

In contrast to $\mathrm{SU}(2)$, several states of an irrep $\Gamma$ can have the same weight. The number of states with the same weight

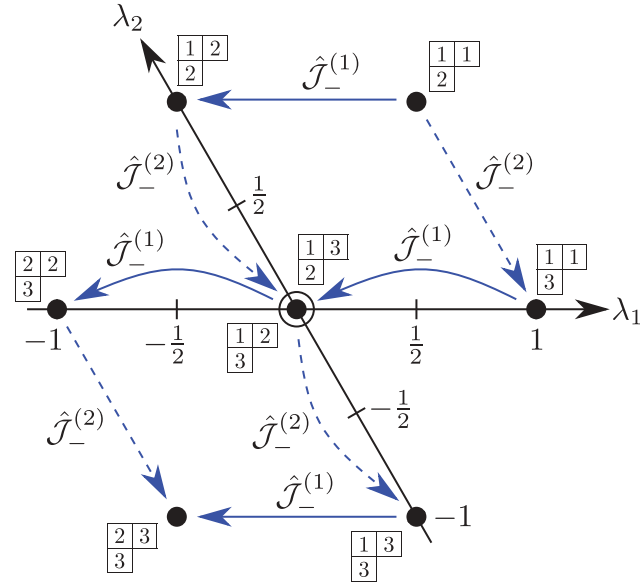

FIG. 11. (Color online) Weight diagram of the SU(3) irrep (nonorthogonal axes chosen to emphasize the symmetric structure of the irrep). Each dot represents a weight; we also indicate the Young tableaux of the corresponding states. The circled dot indicates a weight with inner multiplicity 2 . The blue solid and dashed arrows represent the action of $J_{-}^{(1)}$ and $J_{-}^{(2)}$, respectively.

is called the inner multiplicity of this weight. Consequently, weights are not suitable as the label $\gamma$. Instead, we use one of two equivalent labeling schemes, Young tableaux or GelfandTsetlin patterns (GT patterns).

An $\mathrm{SU}(N)$ Young tableau is a single, contiguous cluster of left-aligned boxes with at most $N$ rows, such that each row is not longer than the one above. Each box of a tableau carries a number between 1 and $N$, inclusive, such that numbers do not decrease from left to right, and numbers increase strictly from top to bottom.

A GT pattern $M$ is a triangular matrix of integers $M_{k, l}(1 \leqslant$ $k \leqslant l \leqslant N)$, commonly written as

$$
M=\left(\begin{array}{cccc}
m_{1, N} & m_{2, N} & \ldots & m_{N, N} \\
m_{1, N-1} & \ldots & m_{N-1, N-1} \\
\ddots & \ddots & \\
m_{1,2} & m_{2,2} \\
& m_{1,1}
\end{array}\right),
$$

which are subject to the so-called betweenness condition,

$$
m_{k, l} \geqslant m_{k, l-1} \geqslant m_{k+1, l} \quad(1 \leqslant k<l \leqslant N) .
$$

Table IV gives examples of equivalent Young tableaux and GT patterns.

Young tableaux and GT patterns are composite labels, $M=$ $(\Gamma, \gamma)$ in short, in the sense that they can play the role of both the irrep label $\Gamma$ and the state label $\gamma$. The shape of a Young tableau (i.e., without the labeling of boxes) determines an irrep $\Gamma$; it corresponds to the top row of a GT pattern, whose $k$ th entry $m_{k, N}$ specifies the number of boxes in the $k$ th row of the Young tableau. The dimension of an irrep $\Gamma$ is equal to the number of valid GT patterns with a given top row. There exists a convenient formula for this number:

$$
\operatorname{dim}(\Gamma)=\prod_{1 \leqslant k<k^{\prime} \leqslant N}\left(1+\frac{m_{k, N}-m_{k^{\prime}, N}}{k^{\prime}-k}\right) .
$$


TABLE IV. Examples of GT patterns and corresponding Young tableaux; the top row of each pattern determines the shape of its respective tableau. These examples have been constructed in such a way that each tableau/pattern contains the examples to its left as a subtableau/subpattern. Note that we usually drop columns of $\mathrm{SU}(N)$ Young tableaux with length $N$ (e.g., the leftmost column in the rightmost tableau); this corresponds to subtracting the entry $m_{N, N}$ from each entry of a GT pattern. The weight of a state can be directly constructed from a GT pattern: Let $\sigma_{l}=\sum_{k=1, \ldots, l} m_{k, l}$ denote the row sums, then $\lambda_{l}^{M}=\left(\sigma_{l+1}-\sigma_{l}\right) / 2$.

$$
\begin{aligned}
& \left(\frac{32}{2}\right) \quad\left(\begin{array}{c}
321 \\
32 \\
2
\end{array}\right) \\
& \left(\begin{array}{cccc}
4 & 3 & 1 & 1 \\
\hline 3 & 2 & 1 \\
3 & 2 \\
2 &
\end{array}\right)
\end{aligned}
$$

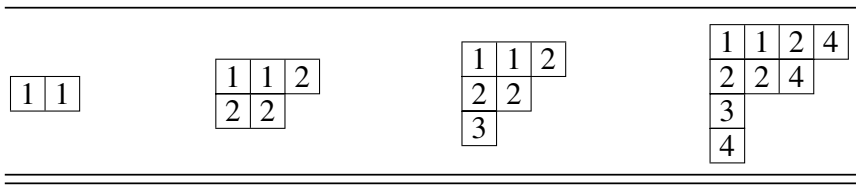

The GT labeling scheme has the advantage that for any of the generators $\hat{\mathcal{J}}^{a} \in\left\{\hat{\mathcal{J}}_{z, \pm}^{(l)}\right\}$, the corresponding matrix elements $\Gamma_{\gamma^{\prime}, \gamma}^{a}$ [Eq. (A3)] within the irrep $\Gamma$ are known explicitly, given by a complicated formula worked out by Gelfand and Tsetlin. ${ }^{33}$

A further ingredient to $\mathrm{SU}(N)$ representation theory is the decomposition of a tensor product $\Gamma_{1} \otimes \Gamma_{2}$ of two irreps into a direct sum of irreps [see Eq. (10)]. For $\mathrm{SU}(N)$, this trick is accomplished by the Littlewood-Richardson rule, which is beyond the scope of this introduction. It produces equations such as

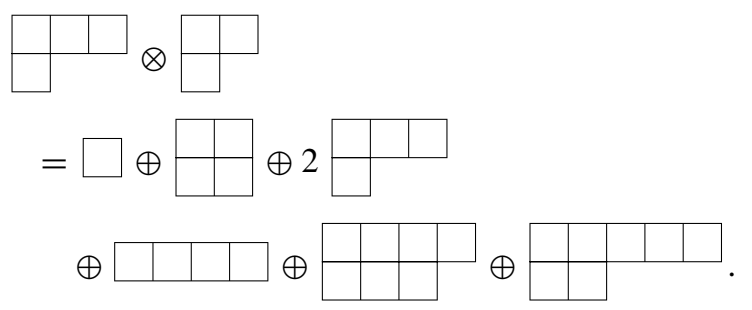

The number of times a particular irrep occurs on the righthand side is called its outer multiplicity; for $\mathrm{SU}(N)$, it is $>1$ in general. The particular basis transformation affecting this decomposition is described by Clebsch-Gordan coefficients [see Eq. (11)]; Ref. 16 presents a numerical algorithm for computing them for any $N$, according to the following strategy.

To determine the $\mathrm{CG}$ coefficients, we need to construct explicitly the state of an irrep $\Gamma$ from the decomposition of $\Gamma_{1} \otimes \Gamma_{2}$. To do that we make an ansatz for it in terms of direct product states,

$$
|\Gamma, \gamma\rangle=\sum_{\gamma_{1}, \gamma_{2}}\left(\Gamma_{1}, \gamma_{1} ; \Gamma_{2}, \gamma_{2} \mid \Gamma, \gamma\right)\left|\Gamma_{1}, \gamma_{1}\right\rangle\left|\Gamma_{2}, \gamma_{2}\right\rangle,
$$

where for the sake of simplicity, we assumed no outer multiplicity [see Eq. (11)]. First, we find the Clebsch-Gordan coefficients of the highest-weight state of $\Gamma$ from the requirement that it is annihilated by all $\hat{\mathcal{J}}_{+}^{(l)}$. Inserting the ansatz into Eq. (A4) generates a system of linear equations; its solution are the highest-weight $\mathrm{CG}$ coefficients. Then, we proceed to the desired state by applying $\hat{\mathcal{J}}_{-}^{(l)}$ operators on the expansion of the highest-weight state, using the matrix elements defined
TABLE V. Organization of the impurity states into multiplets. The $2^{3}=8$ states are organized into four multiplets, each characterized

\begin{tabular}{|c|c|c|c|c|c|}
\hline Multiplets & States & $Q$ & $F$ & Energy & Degeneracy \\
\hline 1 & $\begin{aligned}|1\rangle & =|0\rangle \\
|2\rangle & =d_{1}^{\dagger}|0\rangle\end{aligned}$ & $-3 / 2$ & $\bullet$ & 0 & 1 \\
\hline 2 & $\left.\begin{array}{rl}|3\rangle & =d_{2}^{\dagger}|0\rangle \\
|4\rangle & =d_{3}^{\dagger}|0\rangle\end{array}\right\}$ & $-1 / 2$ & $\square$ & $\varepsilon_{d}$ & 3 \\
\hline 3 & $\left.\begin{array}{l}|6\rangle=d_{1}^{\dagger} d_{3}^{\dagger}|0\rangle \\
|7\rangle=d_{2}^{\dagger} d_{3}^{\dagger}|0\rangle\end{array}\right\}$ & $1 / 2$ & $\square$ & $2 \varepsilon_{d}+U$ & 3 \\
\hline 4 & $|8\rangle=d_{1}^{\dagger} d_{2}^{\dagger} d_{3}^{\dagger}|0\rangle$ & $3 / 2$ & $\bullet$ & $3 \varepsilon_{d}+3 U$ & 1 \\
\hline
\end{tabular}
by a set of quantum numbers $(Q, F)$. States within a multiplet are degenerate in energy.

in Eq. (A3). This procedure requires the calculation of all CG coefficients of states inside of $\Gamma$ with higher weight than $|\Gamma, \gamma\rangle$. Thus, one usually determines all CG coefficients of a given $\Gamma$ in one go. Irreps $\Gamma$ with outer multiplicity $>1$ need special attention, but the principle algorithm remains the same.

Now consider a quantum chain model involving $N$ creation and annihilation operators per site, $f_{\alpha}^{[n] \dagger}$ and $f_{\alpha}^{[n]}$ for site $n$, with $\alpha=1, \ldots, N$. For a given site $n$, consider the set of operators,

$$
\hat{J}^{a,[n]} \equiv \sum_{\alpha, \beta=1}^{N} f_{\alpha}^{[n]^{\dagger}}\left(J^{a}\right)_{\alpha, \beta} f_{\beta}^{[n]},
$$

with the matrices $J^{a}$ taken as the defining representation of $\mathrm{SU}(N)$ [Eq. (A2)]. The $\hat{J}^{a,[n]}$ satisfy the same commutation relations as the $\mathrm{SU}(N)$ generators $\hat{\mathcal{J}}^{a}$ and hence generate an operator representation of $\mathrm{SU}(N)$ on the Fock space of site $n$. The action of these operators partitions this Fock space into a direct sum of irreps. For example, Table V specifies these irreps explicitly for the case of SU(3).

The Fock space of the full chain is the direct product of the Fock spaces of each site. Correspondingly, $\hat{J}^{a}=\sum_{\oplus n} \hat{J}^{a,[n]}$ generates an operator representation of $\operatorname{SU}(N)$ on the full chain of length $n$, which can be decomposed into a direct sum of irreps by iterative use of the Littlewood-Richardson rule and Clebsch-Gordan coefficients.

The Hamiltonian $\hat{H}$ for the full chain has $\mathrm{SU}(N)$ symmetry if it commutes with all generators $\hat{J}^{a}$. When the Hamiltonian is expressed in the Fock space basis just mentioned, consisting of a direct sum of $\mathrm{SU}(N)$ irreps, it is block diagonal, with each block containing matrix elements only between states transforming according to a given $\mathrm{SU}(N)$ irrep.

Diagonalizing such blocks, or more generally, calculating matrix elements of operators, is expedited by using the Wigner-Eckart theorem. To this end, one needs to group operators in Fock space into operator multiplets (sometimes called irreducible tensor operators). An operator multiplet transforming according to the irrep $\Gamma$ is a set of operators $\hat{O}_{\Gamma, \gamma}$ that satisfy the relations,

$$
\left[\hat{J}^{\alpha}, \hat{O}_{\Gamma, \gamma}\right]=\sum_{\gamma^{\prime}} \Gamma_{\gamma^{\prime}, \gamma}^{\alpha} \hat{O}_{\Gamma, \gamma^{\prime}}
$$


For example, the set of all generators $\hat{J}^{\alpha}$ constructed in Eq. (A10) spans an operator multiplet, acting only on site $n$ and transforming according to the adjunct representation of $\operatorname{SU}(N)$, which has dimension $N^{2}-1$. [For SU(3), this is the irrep $\square$ listed in Table I].

A convenient way to explicitly construct an operator multiplet associated with a given site $n$ is to find its highestweight operator by guessing or repeated application of raising operators, and then produce the other operators in the multiplet by applying lowering operators, using Eq. (A11). Table I gives some examples of SU(3) operator multiplets constructed in this manner.

The tensor product of two operator multiplets, each acting individually on a separate site, can be decomposed into a direct sum of two-site operator multiplets, again using the Richardson-Littlewood rule and Clebsch-Gordan coefficients. However, one often finds that extending a single-site operator multiplet to two sites by taking its tensor product with the identity operator [transforming according to the trivial $\mathrm{SU}(N)$ representation] is enough, so a complicated decomposition can be avoided in most cases. For more than two sites, this procedure is applied iteratively.

\section{APPENDIX B: MULTIPLETS WITHIN THE ANDERSON MODEL WITH $U_{Q}(1) \times S U_{F}(3)$ SYMMETRY}

In this Appendix we illustrate in more detail how the general concepts presented in Appendix A can be applied to our case, when $U_{Q}(1) \times S U_{F}(3)$ symmetry is used. We first construct the lowering/raising operators in the Fock space by using Eq. (A10). Explicitly, for SU(3) we shall need explicitly six generators,

$$
\begin{aligned}
& \hat{J}_{+}^{(1)}=d_{1}^{\dagger} d_{2}, \quad \hat{J}_{+}^{(2)}=d_{2}^{\dagger} d_{3}, \\
& \hat{J}_{-}^{(1)}=d_{2}^{\dagger} d_{1}, \quad \hat{J}_{-}^{(2)}=d_{3}^{\dagger} d_{2}, \\
& \hat{J}_{z}^{(1)}=\frac{1}{2}\left(d_{1}^{\dagger} d_{1}-d_{2}^{\dagger} d_{2}\right), \quad \hat{J}_{z}^{(2)}=\frac{1}{2}\left(d_{2}^{\dagger} d_{2}-d_{3}^{\dagger} d_{3}\right) .
\end{aligned}
$$

The initial impurity states can be constructed and organized into four SU(3) multiplets relatively easily. They are presented in Table $\mathrm{V}$. The highest weight states $|\Gamma, \gamma=\Gamma\rangle$ can be found by requiring that both $\hat{J}_{+}^{(1)}$ and $\hat{J}_{+}^{(2)}$ annihilate them. Further states within the multiplet are then obtained by acting with $\hat{J}_{-}^{(1)}$ and $\hat{J}_{-}^{(2)}$, and comparing to Eq. (A3). Notice that the ClebschGordan coefficients do depend on the particular choice of basis, and therefore, to make use of the Wigner-Eckart theorem, every multiplet must be constructed to conform with the same choice of basis as the Clebsch-Gordan coefficients. This choice of basis is implicitly contained in the matrix elements $\left(\Gamma_{ \pm}^{(l)}\right)_{\gamma \gamma^{\prime}}$. In the open access flexible NRG code ${ }^{13}$ we used the conventions of Ref. 16, for which these matrix elements are explicitly given in Ref. 33.

To use the Wigner-Eckart theorem, we need to organize operators into operator multiplets. A few examples of these were presented in Table I. Let us discuss here the specific case of the spin operator, forming an eight-dimensional multiplet, which transforms according to the $\square$ representation. A similar procedure applies to the hopping or the superconducting operators.

We can quickly guess that the highest weight operator is $d_{1}^{\dagger} d_{3}$ since it commutes with both $\hat{J}_{+}^{(1)}$ and $\hat{J}_{+}^{(2)}$ [see Eq. (A11)]. This operator thus corresponds to the \begin{tabular}{|l|l|l|l|l|l}
\hline & 1 & state. Now, by \\
\hline & & sta
\end{tabular} applying the lowering operators (i.e., forming commutators with them) as indicated in Fig. 11, we can generate all the other operators that form the multiplet. ${ }^{16}$ In this procedure one needs again the explicit form of the corresponding matrix elements, $\left(\Gamma_{ \pm}^{(l)}\right)_{\gamma \gamma^{\prime}}$ to fix the proper phase/sign of the basis states and their normalization.

We remark that particular care must be taken in cases where a weight has an inner multiplicity, as for the members \begin{tabular}{ll}
1 & 3 \\
\hline
\end{tabular} and \begin{tabular}{|l|l|l|l}
\hline 1 & 2 & of the spin operator multiplet. There one needs to take \\
\hline & &
\end{tabular} the correct linear combination of the corresponding operators to transform according to $\left(\Gamma_{ \pm}^{(l)}\right)_{\gamma \gamma^{\prime}}$ [see Eq. (A11)].

\section{APPENDIX C: RECURSION FORMULAS}

In this section we shall detail how the recursive relations [Eqs. (16)-(19)] were derived. The general procedure is based on the Wigner-Eckart theorem and using sum rules satisfied by the Clebsch-Gordan coefficients. We first derive the recursion relation for the irreducible matrix element of the hopping Hamiltonian. The general expression of the hopping matrix element at iteration $n$ reads

$$
\alpha_{u}\left\langle u, \Gamma_{u}, \gamma_{u}\left|\tau_{n-1, n}\right| v, \Gamma_{v}, \gamma_{v}\right\rangle_{\alpha_{v}}^{[n]}=\sum_{a}\left[h_{a}^{[n-1]} \sum_{\gamma_{a}} \alpha_{u}\left\langle u, \Gamma_{u}, \gamma_{u}\left|C_{a, \Gamma_{a}, \gamma_{a}}^{[n-1]}\left(C_{a, \Gamma_{a}, \gamma_{a}}^{[n]}\right)^{\dagger}\right| v, \Gamma_{v}, \gamma_{v}\right\rangle_{\alpha_{v}}^{[n]}+\text { H.c. }\right] \text {. }
$$

Here the multiplets, $\left|u, \Gamma_{u}, \gamma_{u}\right\rangle_{\alpha_{i}}^{[n]}$ and $\left|v, \Gamma_{v}, \gamma_{v}\right\rangle_{\alpha_{v}}^{[n]}$, were constructed in terms of the block multiplet $\left|i, \Gamma_{i}, \gamma_{i}\right\rangle^{[n-1]}$ and local multiplet $\left|\mu, \Gamma_{\mu}^{\text {loc }}, \gamma_{\mu}^{\text {loc }}\right\rangle$ at iteration $n-1$ using Eq. (11). Next we exploit the locality of the $C_{a, \Gamma_{a}, \gamma_{a}}^{[n]}$, that is, that the operator $C_{a, \Gamma_{a}, \gamma_{a}}^{[n]}$ acts only on local states $\mu$, while $\left(C_{a, \Gamma_{a}, \gamma_{a}}^{[n-1]}\right)^{\dagger}$ acts on the block states $i$. The matrix element of Eq. (C1) then becomes

$$
\begin{aligned}
\alpha_{u}\left\langle u, \Gamma_{u}, \gamma_{u}\left|\tau_{n-1, n}\right| v, \Gamma_{v}, \gamma_{v}\right\rangle_{\alpha_{v}}^{[n]}= & \sum_{a}\left[h_{a}^{[n-1]} \operatorname{sgn}\left(C_{a}, \mu\right) \sum_{\gamma_{a}} \sum_{\gamma_{i}, \gamma_{\mu}^{\text {loc }}, \gamma_{j} \gamma_{v}^{\text {loc }}}\left(\Gamma_{\mu}^{\mathrm{loc}}, \gamma_{\mu}^{\mathrm{loc}} ; \Gamma_{i}, \gamma_{i} \mid \Gamma_{u}, \gamma_{u}\right)_{\alpha_{u}}^{*}\left(\Gamma_{v}^{\mathrm{loc}}, \gamma_{v}^{\mathrm{loc}} ; \Gamma_{j}, \gamma_{j} \mid \Gamma_{u}, \gamma_{u}\right)_{\alpha_{v}}\right. \\
& \left.\times\left\langle i, \Gamma_{i}, \gamma_{i}\left|C_{a, \Gamma_{a}, \gamma_{a}}^{[n-1]}\right| j, \Gamma_{j}, \gamma_{j}\right\rangle^{[n-1]}\left\langle v, \Gamma_{v}^{\mathrm{loc}}, \gamma_{v}^{\mathrm{loc}}\left|C_{a, \Gamma_{a}, \gamma_{a}}^{[n]}\right| \mu, \Gamma_{\mu}^{\mathrm{loc}}, \gamma_{\mu}^{\mathrm{loc}}\right\rangle+\text { H.c. }\right]
\end{aligned}
$$


Here the sign function $\operatorname{sgn}\left(C_{a}, \mu\right)= \pm 1$ arises as we commute the local state $\mu$ over the operator $C^{[n-1]}$. If the hopping operator $C$ is fermionic and the local state contains an odd number of fermions, then the sign is negative; otherwise it is positive. Now we can use the Wigner-Eckart theorem, Eq. (13), and express the matrix elements of the creation/annihilation operators in terms of their reduced matrix elements. By doing that, we immediately recover the result (16) with

$$
\begin{aligned}
D(a, \alpha, \beta ; u, v)= & \operatorname{sgn}(a, \mu) \sum_{\gamma_{c}} \sum_{\gamma_{i}, \gamma_{\mu}^{\mathrm{loc}}} \sum_{\gamma_{j}, \gamma_{v}^{\mathrm{loc}}}\left(\Gamma_{\mu}^{\mathrm{loc}}, \gamma_{\mu}^{\mathrm{loc}} ; \Gamma_{i}, \gamma_{i} \mid \Gamma_{u}, \gamma_{u}\right)_{\alpha_{u}}^{*}\left(\Gamma_{v}^{\mathrm{loc}}, \gamma_{v}^{\mathrm{loc}} ; \Gamma_{j}, \gamma_{j} \mid \Gamma_{u}, \gamma_{u}\right)_{\alpha_{v}} \\
& \times\left(\Gamma_{a}, \gamma_{a} ; \Gamma_{j}, \gamma_{j} \mid \Gamma_{i}, \gamma_{i}\right)_{\alpha}^{*}\left(\Gamma_{a}, \gamma_{a} ; \Gamma_{\mu}^{\mathrm{loc}}, \gamma_{\mu}^{\mathrm{loc}} \mid \Gamma_{v}^{\mathrm{loc}}, \gamma_{\nu}^{\mathrm{loc}}\right)_{\beta} .
\end{aligned}
$$

A slightly different analysis can be done for block operators. First we notice that we can "invert" the Wigner-Eckart theorem, Eq. (13), using the completeness of the Clebsch-Gordan coefficients, and express the reduced matrix elements instead as

$$
\langle u\|A\| v\rangle_{\beta}^{[n]}=\sum_{\gamma_{A}, \gamma_{v}} \alpha_{u}\left\langle u, \Gamma_{u}, \gamma_{u}\left|A_{\Gamma_{A}, \gamma_{A}}\right| v, \Gamma_{v}, \gamma_{v}\right\rangle_{\alpha_{v}}^{[n]}\left(\Gamma_{A}, \gamma_{A} ; \Gamma_{v}, \gamma_{v} \mid \Gamma_{u}, \gamma_{u}\right)_{\beta} .
$$

As a next step, we need to express the matrix element $\alpha_{u}\left\langle u, \Gamma_{u}, \gamma_{u}\left|A_{\Gamma_{A}, \gamma_{A}}\right| v, \Gamma_{v}, \gamma_{v}\right\rangle_{\alpha_{v}}^{[n]}$ in Eq. (C4) in terms of the irreducible matrix elements of the operator at iteration $n-1$. Here we follow the same strategy as in the case of the hopping operator: We expand first the states using Eq. (D2), while keeping in mind that the operator acts only in the block states sector, and then use the Wigner-Eckart theorem (13) for the operator's matrix elements. By doing that we find

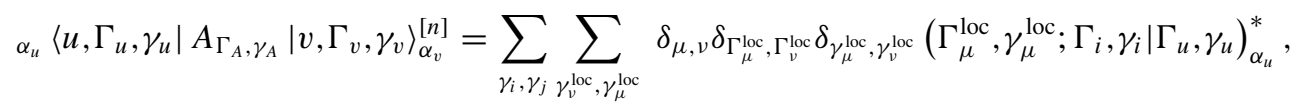

$$
\begin{aligned}
& \left(\Gamma_{v}^{\mathrm{loc}}, \gamma_{v}^{\mathrm{loc}} ; \Gamma_{j}, \gamma_{j} \mid \Gamma_{v}, \gamma_{v}\right)_{\alpha_{v}} \operatorname{sgn}(A, \mu) \sum_{\alpha}\left(\Gamma_{A}, \gamma_{A} ; \Gamma_{j}, \gamma_{j} \mid \Gamma_{i}, \gamma_{i}\right)_{\alpha}^{*}\langle i\|A\| j\rangle_{\alpha}^{[n-1]}
\end{aligned}
$$

Plugging Eq. (C5) in Eq. (C4) we recover the result for the block operator stated in Eq. (18) with the coefficient $F$ defined as

$$
\begin{aligned}
F(\alpha, \beta ; u, v)= & \operatorname{sgn}(A, \mu) \sum_{\gamma_{\mu}^{\text {loc }}} \sum_{\gamma_{i}, \gamma_{j}} \sum_{\gamma_{A}, \gamma_{\tilde{j}}}\left(\Gamma_{\mu}^{\mathrm{loc}}, \gamma_{\mu}^{\text {loc }} ; \Gamma_{i}, \gamma_{i} \mid \Gamma_{u}, \gamma_{u}\right)_{\alpha_{u}}^{*}\left(\Gamma_{\mu}^{\mathrm{loc}}, \gamma_{\mu}^{\text {loc }} ; \Gamma_{j}, \gamma_{j} \mid \Gamma_{v}, \gamma_{v}\right)_{\alpha_{v}} \\
& \times\left(\Gamma_{A}, \gamma_{A} ; \Gamma_{j}, \gamma_{j} \mid \Gamma_{i}, \gamma_{i}\right)_{\alpha}^{*}\left(\Gamma_{A}, \gamma_{A} ; \Gamma_{v}, \gamma_{v} \mid \Gamma_{u}, \gamma_{u}\right)_{\beta} .
\end{aligned}
$$

A similar analysis can be done in the case of a "local operator," giving the final expression for the coefficient $K$ entering Eq. (19):

$$
\begin{aligned}
K(\alpha, \beta ; u, v)= & \sum_{\gamma_{i}} \sum_{\gamma_{\mu}^{\mathrm{loc}}, \gamma_{v}^{\mathrm{loc}} \gamma_{A}, \gamma_{v}}\left(\Gamma_{\mu}^{\mathrm{loc}}, \gamma_{\mu}^{\mathrm{loc}} ; \Gamma_{i}, \gamma_{i} \mid \Gamma_{u}, \gamma_{u}\right)_{\alpha_{u}}^{*}\left(\Gamma_{v}^{\mathrm{loc}}, \gamma_{v}^{\mathrm{loc}} ; \Gamma_{i}, \gamma_{i} \mid \Gamma_{v}, \gamma_{v}\right)_{\alpha_{v}} \\
& \times\left(\Gamma_{A}, \gamma_{A} ; \Gamma_{v}^{\mathrm{loc}}, \gamma_{v}^{\mathrm{loc}} \mid \Gamma_{\mu}^{\mathrm{loc}}, \gamma_{\mu}^{\mathrm{loc}}\right)_{\alpha}^{*}\left(\Gamma_{A}, \gamma_{A} ; \Gamma_{v}, \gamma_{v} \mid \Gamma_{u}, \gamma_{u}\right)_{\beta} .
\end{aligned}
$$

\section{APPENDIX D: CONNECTION TO THE MATRIX PRODUCT STATES APPROACH}

It is well known that the states constructed within the NRG framework can be viewed as matrix product states (MPS). ${ }^{34}$ Moreover, it has been shown recently that non-Abelian symmetries can be incorporated into the construction of MPS. ${ }^{15,35}$ In this Appendix we briefly review how this can be done in the context of NRG. We start with the simple observation that the Hamiltonian of a chain of length $N, H_{N}$ acts on the Hilbert space spanned by a basis constructed from local multiplets:

$$
\left\{\left|\mu_{0}, \Gamma_{\mu_{0}}^{\mathrm{loc}}, \gamma_{\mu_{0}}^{\mathrm{loc}} ; \mu_{1}, \Gamma_{\mu_{1}}^{\mathrm{loc}}, \gamma_{\mu_{1}}^{\mathrm{loc}} ; \ldots ; \mu_{N}, \Gamma_{\mu_{N}}^{\mathrm{loc}}, \gamma_{\mu_{N}}^{\mathrm{loc}}\right\rangle\right\}
$$

The dimension of this basis set is $d_{0} d^{N}$, with $d_{0}$ the dimension of $\mathcal{H}_{0}$, and $d$ is the dimension of the Hilbert space at sites along the Wilson chain (see Table V). For the SU(3) Anderson model we have four multiplets on each site, and $d_{0}=d=8$.

Let us now assume that we have constructed somehow some block states $i$, which span the relevant part of the Hilbert space of a chain with $n-1$ sites. The number of these states, $D$ is, of course, much less than the total number of states within this block, which would be of the order of $\sim d^{n}$. We can then use these states to express the appropriate (relevant) states for a chain of $n$ sites as

$$
\left|\tilde{i}, \Gamma_{\tilde{i}}, \gamma_{\tilde{i}}\right\rangle_{\alpha_{\tilde{i}}}^{[n]}=\sum_{i, \Gamma_{i}, \gamma_{i}}^{\sim} \sum_{\mu, \Gamma_{\mu}^{[\mathrm{loc}]}, \gamma_{\mu}^{[l o c]}}\left(P_{\Gamma_{i} \gamma_{i}, \Gamma_{\tilde{i}} \gamma_{i} ; \alpha_{\tilde{i}}}^{\left.\left[\Gamma_{\tilde{i}}^{\mathrm{loc}}\right)_{i \tilde{i}}^{\text {loc }}\right]}\left|\mu, \Gamma_{\mu}^{\mathrm{loc}}, \gamma_{\mu}^{\mathrm{loc}}\right\rangle \otimes\left|i, \Gamma_{i}, \gamma_{i}\right\rangle^{[n-1]}\right.
$$

Here $P$ is some projector that generates the relevant block of multiplets $\{|\tilde{i}\rangle\}$ from the block multiplets $\{|i\rangle\}$ by adding some local states $\{|\mu\rangle\}$. The tilde in the sum indicates that only a number $D$ of multiplets are kept at each iteration. In the presence of 
symmetries, the matrices $P$ can be factorized into products of reduced matrix elements and Clebsch-Gordan coefficients, as

$$
\left(P_{\Gamma_{i} \gamma_{i}, \Gamma_{i} \gamma_{i}, \alpha_{i}}^{\left[\Gamma_{i}^{\text {loc }}\right.}\right)_{i \tilde{i}}^{\text {loc }}=\left(A_{\Gamma_{i} \Gamma_{i}}^{\left[\Gamma_{\mu}^{\text {loc }}\right]}\right)_{i \tilde{i}}^{[\mu]}\left(C_{\Gamma_{i} \Gamma_{i} ; \alpha_{i}}^{\left[\Gamma_{i}^{\text {loc }}\right]}\right)_{\gamma_{i} \gamma_{i}}^{\left[\gamma_{\mu}^{\text {loc }}\right]},
$$

with

$$
\left(C_{\Gamma_{i} \Gamma_{\tilde{i}} ; \alpha_{\tilde{i}}}^{\left[\Gamma_{\tilde{i}}^{\mathrm{loc}}\right]}\right)_{\gamma_{i} \gamma_{i}}^{\left[\gamma_{\mu}^{\text {loc }}\right]}=\left(\Gamma_{i}, \gamma_{i} ; \Gamma_{\mu}^{\mathrm{loc}}, \gamma_{\mu}^{\mathrm{loc}} \mid \Gamma_{\tilde{i}}, \gamma_{\tilde{i}}\right)_{\alpha_{\tilde{i}}^{*}} .
$$

Here the key observation is that the reduced matrix elements $\left(A_{\Gamma_{i} \Gamma_{\tilde{i}}}^{\left[\Gamma_{\mu}^{\mathrm{loc}}\right]}\right)_{i \tilde{i}}^{[\mu]}$ can also be thought of as the matrix elements of some irreducible operators, labeled by $\Gamma_{\mu}^{\mathrm{loc}}$.

Repeating the iteration procedure multiplets describing the full chain of size $N$ can be constructed this way as a matrix product state (summation of repeated indices is implicitly assumed),

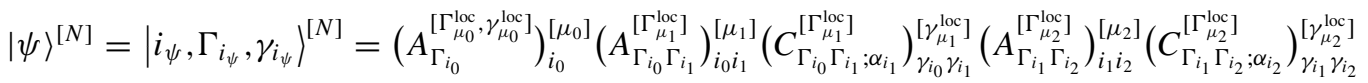

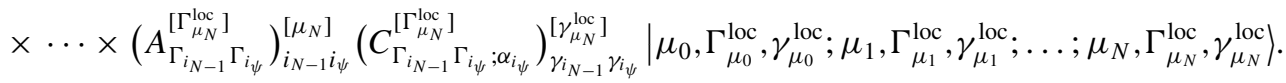

Note that the index structure that arises here implies matrix multiplication not only for the $A$ matrices of reduced matrix elements, but also for the $C$ matrices of Clebsch-Gordan coefficients. This MPS formulation can be used either to implement standard Wilsonian truncation (as done in Ref. 15), or, alternatively, to proceed variationally, as done in the density-matrix renormalization group (DMRG) ${ }^{36}$ whose use for Wilson chains was explored in Refs. 34 and 37. In the latter case, one views the $A$ matrices as a set of variational parameters that need to be optimized according to some criteria. To find the optimal approximation for the ground state, for example, we look for the corresponding MPS which minimizes the total energy,

$$
E=\frac{\left\langle\psi\left|H_{N}\right| \psi\right\rangle^{[N]}}{\langle\psi \mid \psi\rangle^{[N]}} .
$$

This variational problem can be converted into a generalized eigenvalue problem and solved using an iterative sweepinglike procedure (see Ref. 36 for more technical details). Once the MPS state is found, it is possible to construct the eigenspectrum of the Hamiltonian $H_{N}$ for a fixed $N$. The flow diagram, such as the one presented in Fig. 2, can be obtained by tracing the spectrum of the Hamiltonian with increasing $N$; if the discretization parameter $\Lambda$ is large enough $(\Lambda \gtrsim 2)$, the numerical results thus obtained are essentially equivalent to those using standard Wilsonian truncation. ${ }^{37}$ For more details on how the operators can be treated at the MPS level we direct the reader to the more detailed reviews, Refs. 15 and 36.

${ }^{1}$ P. W. Anderson, Phys. Rev. 124, 41 (1961).

${ }^{2}$ N. E. Bickers, Rev. Mod. Phys. 59, 845 (1987).

${ }^{3}$ C. Honerkamp and W. Hofstetter, Phys. Rev. Lett. 92, 170403 (2004).

${ }^{4}$ A. Rapp, G. Zárand, C. Honerkamp, and W. Hofstetter, Phys. Rev. Lett. 98, 160405 (2007).

${ }^{5}$ P. Jarillo-Herrero, J. Kong, H. S. J. van der Zant, C. Dekker, L. P. Kouwenhoven, and S. De Franceschi, Nature (London) 434, 484 (2005).

${ }^{6}$ A. Makarovski, A. Zhukov, J. Liu, and G. Finkelstein, Phys. Rev. B 75, 241407(R) (2007).

${ }^{7}$ S. Sasaki, S. Amaha, N. Asakawa, M. Eto, and S. Tarucha, Phys. Rev. Lett. 93, 017205 (2004).

${ }^{8} \mathrm{D}$. Goldhaber-Gordon (private communication).

${ }^{9}$ A. Carmi, Y. Oreg, and M. Berkooz, Phys. Rev. Lett. 106, 106401 (2011).

${ }^{10}$ R. Bulla, T. A. Costi, and T. Pruschke, Rev. Mod. Phys. 80, 395 (2008).

${ }^{11}$ K. G. Wilson, Rev. Mod. Phys. 47, 773 (1975). For a recent review of NRG see Ref. 10.

${ }^{12}$ A. I. Tóth, C. P. Moca, Ö. Legeza, and G. Zaránd, Phys. Rev. B 78, 245109 (2008).

${ }^{13}$ The code is accessible at http://www.phy.bme.hu/ $\sim$ dmnrg.
${ }^{14}$ The second generation flexible DM-NRG code, incorporating these changes shall be available for download from December 2012 on.

${ }^{15}$ A. Weichselbaum, Ann. Phys. 327, 2972 (2012).

${ }^{16}$ A. Alex, M. Kalus, A. Huckleberry, and J. von Delft, J. Math. Phys. 52, 023507 (2011). Online resource at http://homepages. physik.uni-muenchen.de/ vondelft/Papers/ClebschGordan/.

${ }^{17}$ Notice also that some equations in Ref. 12 contained typographical errors, which we tried to eliminate here.

${ }^{18}$ This holds for all finite groups and all compact Lie groups as well as for finite dimensional representations of semisimple Lie groups.

${ }^{19} \mathrm{~A}$ local operator always carries an extra label indicating the position along the Wilson chain where it acts.

${ }^{20}$ R. Bulla, A. C. Hewson, and Th. Pruschke, J. Phys.: Condens. Matter 10, 8365 (1998).

${ }^{21}$ W. Hofstetter, Phys. Rev. Lett. 85, 1508 (2000).

${ }^{22}$ R. Peters, Th. Pruschke, and F. B. Anders, Phys. Rev. B 74, 245114 (2006).

${ }^{23}$ A. Weichselbaum and J. von Delft, Phys. Rev. Lett. 99, 076402 (2007).

${ }^{24}$ Some care is needed with the usage of the Hermitian conjugation sign. In certain cases, one may call an operator multiplet, $A=$ $F^{\dagger}$, for example. In this case, $\left(\left(F^{\dagger}\right)_{\gamma_{F}}\right)^{\dagger}$ is not necessarily equal to $F_{\gamma_{F}}$. 
${ }^{25}$ F. B. Anders and A. Schiller, Phys. Rev. Lett. 95, 196801 (2005).

${ }^{26}$ F. B. Anders and A. Schiller, Phys. Rev. B 74, 245113 (2006).

${ }^{27}$ A. Rapp and A. Rosch, Phys. Rev. A 83, 053605 (2011).

${ }^{28} \mathrm{As} Q$ is semi-integer in the code we have used $2 Q$ as the charge quantum numbers.

${ }^{29}$ Notice that the Clebsch-Gordan coefficients are basis dependent, and to use the Wigner-Eckart theorem, for example, one always needs to make sure that the basis operators within an operator multiplet transform identically with the states which were used to compute the Clebsch-Gordan coefficients.

${ }^{30}$ A. C. Hewson, J. Phys.: Condens. Matter 18, 1815 (2006).

${ }^{31}$ G. Klingschat and C. Honerkamp, Phys. Rev. B 82, 094521 (2010).
${ }^{32}$ L. Gaudreau, S. A. Studenikin, A. S. Sachrajda1, P. Zawadzki, A. Kam, J. Lapointe, M. Korkusinski, and P. Hawrylak, Phys. Rev. Lett. 97, 036807 (2006).

${ }^{33}$ I. M. Gelfand and M. L. Tsetlin, Dokl. Akad. Nauk SSSR 71, 825 (1950); 71, 1017 (1950); reprinted in I. M. Gelfand, R. A. Minlos, and Z. Ya. Shapiro, Representations of the Rotation and Lorentz Group (Pergamon, New York, 1963).

${ }^{34}$ A. Weichselbaum, F. Verstraete, U. Schollwöck, J. I. Cirac, and Jan von Delft, Phys. Rev. B 80, 165117 (2009).

${ }^{35}$ S. Singh, R. N. C. Pfeifer, and G. Vidal, Phys. Rev. A 82, 050301 (2010).

${ }^{36}$ U. Schollwöck, Ann. Phys. 326, 96 (2011).

${ }^{37}$ H. Saberi, A. Weichselbaum, and J. von Delft, Phys. Rev. B 78, 035124 (2008). 